\title{
铁催化氢化反应研究进展
}

\author{
郭 娜朱守非* \\ (南开大学化学学院元素有机化学研究所 元素有机国家重点实验室 天津 300071)
}

\begin{abstract}
摘要 过渡金属催化不饱和有机化合物(如烯烃、炔烃、醛、酮、亚胺等)的氢化反应具有原子经济性高、操作简单、 清洁绿色等优点, 是最重要的有机反应之一, 一直是研究的重点和热点, 并在工业生产中得到广泛应用. 目前氢化反 应最常用的催化剂主要基于铑、钓、铱、钯等贵金属, 这些金属面临着资源枯竭、价格昂贵、重金属残留等问题. 铁 储量丰富、价格便宜、环境友好, 发展铁催化的氢化反应符合可持续发展化学的要求, 近年来受到广泛关注. 以铁催化 剂为主线, 系统综述了均相和非均相铁催化的氢化反应.
\end{abstract}

关键词 铁催化剂; 氢化反应; 不饱和有机化合物.

\section{Iron-Catalyzed Hydrogenation Reactions}

\author{
Guo, $\mathrm{Na}$ Zhu, Shoufei* \\ (State Key Laboratory and Institute of Elemento-Organic Chemistry, College of Chemistry, \\ Nankai University, Tianjin 300071)
}

\begin{abstract}
The transition-metal-catalyzed hydrogenation of unsaturated organic compounds including alkenes, alkynes, aldehydes, ketones, and imines, is one of the most important organic transformations for both academic researches and industrial applications. The most popular catalysts for hydrogenation reactions are based on precious metals, such as rhodium, ruthenium, iridium, and palladium. The exhausting resource, the increasing high price, and the toxicity of the precious metals restrict their future applications. Iron is the most abundant transition metal in the earth crust and therefore the cheapest one with excellent environmental benign characters, is considered as an ideal replacement of the precious metal catalysts for hydrogenation reactions. This review paper introduced the newest achievements in the study of iron-catalyzed hydrogenation reactions in both homogenous and heterogeneous systems.
\end{abstract}

Keywords iron catalyst; hydrogenation; unsaturated organic compound

过渡金属催化不饱和有机化合物(如烯烃、炔烃、 醛、酮、亚胺等)的氢化反应具有原子经济性高、操作 简单、清洁绿色等优点, 是最重要的有机反应之一, 一 直是研究的重点和热点, 并在工业生产中得到广泛应 用 ${ }^{[1]}$. 非均相催化剂, 如负载钯和 Raney 镍, 曾经在氢 化反应中占据主导地位; 1966 年以来, 得益于 Wilkinson 催化剂的发明 ${ }^{[2]}$, 均相催化氢化得到了长足的发展, 特 别是在不对称催化方面表现出独特的优势 ${ }^{[3]}$. 美国化学 家 Knowles 和日本化学家 Noyori 就是由于在不对称催 化氢化反应研究中的卓越贡献而荣获 2001 年度诺贝尔 化学奖 ${ }^{[4]}$. 目前工业化的不对称催化反应中, 有一半以
上是过渡金属催化的氢化反应 ${ }^{[5]}$. 虽然过渡金属催化的 氢化反应研究已经取得辉煌成就, 但是该反应的有效催 化剂绝大多数是基于铑、钉、铱、钯等贵金属, 由于这 些金属储量稀少，价格昂贵，而且很多还有毒性，对环 境造成危害，在工业应用中也存在金属残留等问题，严 重制约了其未来的发展. 铁是地壳中含量最高的过渡金 属, 也是最便宜的金属, 具有很好的生物兼容性. 正是 由于铁的这些特点, 使得铁催化剂成为人们努力发展的 新一代催化剂 ${ }^{[6]}$. 最近几年, 铁催化的氢化反应逐渐成 为研究热点. 目前已经有一些关于铁催化氢化的综述文 章 ${ }^{[7]}$, 但存在以下局限: (1)大多综述文献是从某一个侧

* E-mail:sfzhu@nankai.edu.cn

Received February 26, 2015; revised March 28, 2015; published online April 1, 2015.

Project supported by the National Natural Science Foundation of China (Nos. 21222205, 21172113, 21421062), the National Basic Research Program of China (973 Program, No. 2011CB808600), the “111” Project (No. B06005) of the Ministry of Education of China, and the National Program for Support of Top-notch Young Professionals.

国家自然科学基金(Nos. 21222205, 21172113, 21421062)、国家重点基础研究发展计划(973 项目, No. 2011CB808600)、教育部“111 计划”(No. B06005) 和国家特支计划资助项目. 
面对铁催化氢化研究成果进行总结, 缺少一个全面介绍 该领域整体发展状况的文章; (2)已有的综述文献大多集 中于均相催化氢化, 对于非均相氢化没有系统的总结, 虽然两种氢化反应从机理上具有相通之处, 也可相互借 鉴; (3)已有的综述大多只包含 2009 年之前的文献, 不能 反映近几年铁催化氢化反应的迅速发展. 针对这些问 题, 本文以铁催化剂的发展为主线, 对铁催化烯烃、炔 烃、醛、酮、亚胺等的氢化反应进行系统综述, 特别介 绍了最新研究成果(文献更新至 2014 年 7 月), 并同时涵 盖了均相催化和非均相催化的相关研究成果, 以利于人 们了解该领域的全貌, 为发展新型高效的铁催化氢化反 应提供借鉴. 最后, 文章还对铁催化氢化反应的机遇和 挑战进行了评述, 以期激发更多研究.

\section{1 铁催化烯烃和炔烃的氢化反应}

铁催化烯烃氢化反应主要有两种机理: (1)零价铁催 化剂通过氧化加成的方式活化 $\mathrm{H}_{2}$, 产生二价铁-负氢中 间体, 然后经历烯烃的插入和还原消除等后续步骤, 完 成催化循环 (Scheme 1, A); (2)铁-负氢催化剂首先与烯 烃发生插入反应, 然后与氢气发生氢解, 完成氢化反应, 在此过程中铁的价态保持不变(Scheme 1, B). 下文就以

A

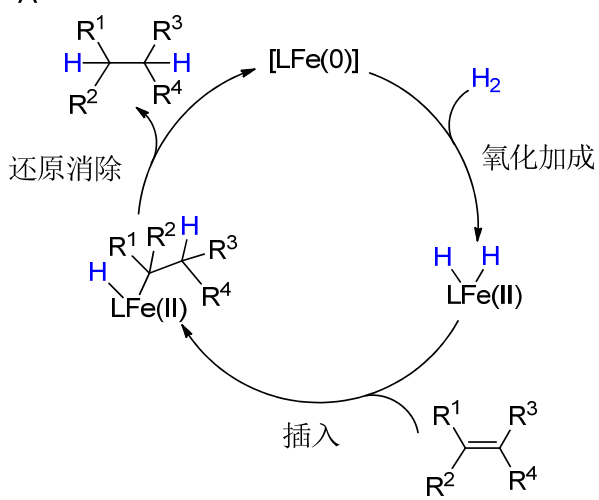

铁催化剂为主线对相关氢化反应进行分类介绍.

\section{1 羰基铁催化剂}

羰基铁是最为稳定和便宜易得的一类 $\operatorname{Fe}(0)$ 化合物, 由于羰基配体在一定条件下可以部分解离, 产生活性位 点，因此可以用作氢化反应的催化剂. 1964 年, Frankel 等 ${ }^{[8]}$ 以 $\mathrm{Fe}(\mathrm{CO})_{5}$ 为催化剂, 实现了亚油酸甲酯(1)的氢化 反应(Scheme 2). 他们采用加热的方法解离羰基配体, 需要很高的反应温度 $\left(180{ }^{\circ} \mathrm{C}\right)$. 这一催化体系的活性不 高, 催化剂用量高达 $10 \mathrm{~mol} \%$, 转化数(单位催化剂转化 的底物数, TON)和转化频率(单位催化剂每小时转化的 底物数, TOF)很低, 产物也比较复杂, 包括部分氢化的 单烯酯 2、完全氢化的硬脂酸甲酯 $\mathbf{3}$ 和各类烯烃异构化 的产物, 其中以单烯酯 2 的含量最高. 机理研究认为, 亚油酸甲酯(1)首先在 $\mathrm{Fe}(\mathrm{CO})_{5}$ 催化下发生异构化, 生成 共轭二烯 4, 随后形成二烯一羰基铁络合物 $\mathbf{5}$, 接下来络 合物 5 被逐步氢化到产物. 由于单烯酯 $\mathbf{2}$ 与铁的络合能 力较共轭二烯大大降低, 造成大部分产物停留在这一 步. 二烯一羰基铁络合物 $\mathbf{5}$ 可以分离鉴定，并在氢化反应 中表现出比 $\mathrm{Fe}(\mathrm{CO})_{5}$ 更高的活性, 说明羰基的解离是能 量较高的步骤. 在相似的反应条件下, $\mathrm{Fe}(\mathrm{CO})_{5}$ 也可以实 现对亚麻酸甲酯这种含有三个 $Z$ 式烯烃的底物的氢化,

B

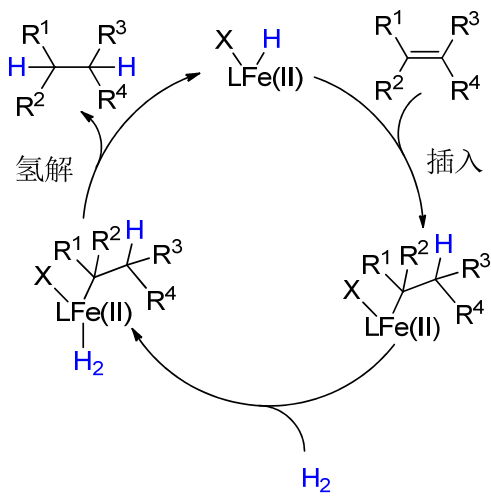

图式 1 铁催化烯烃氢化的两种可能机理

Scheme 1 Two plausible mechanisms of iron-catalyzed hydrogenation of olefins

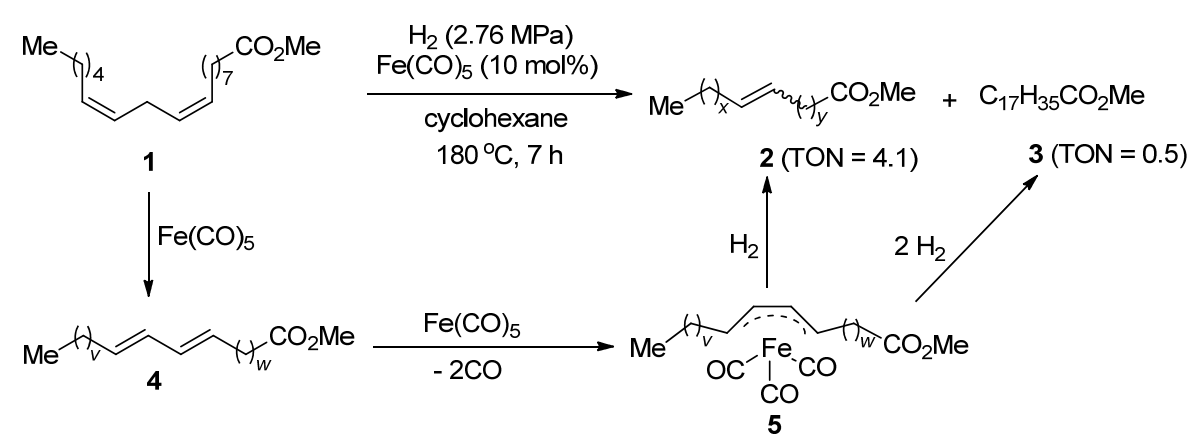

图式 2 铁催化亚油酸甲酯的氢化

Scheme 2 Iron-catalyzed hydrogenation of methyl linoleate 
其结果与亚油酸甲酯的氢化类似 ${ }^{[9]}$.

Cais 和 $\mathrm{Maoz}^{[10]}$ 受到 Frankel 工作的启发, 合成了一 系列双烯-三羰基铁络合物, 并将其用于烯烃的催化氢 化中. 该反应同样需要在较高的温度下 $\left(165{ }^{\circ} \mathrm{C}\right)$ 进行.

Wrighton 等 ${ }^{[11]}$ 利用近紫外光 $(300 \sim 380 \mathrm{~nm})$ 照射的 方式促进 $\mathrm{Fe}(\mathrm{CO})_{5}$ 的羰基的解离, 产生配位不饱和的铁 催化剂, 在温和的条件下 $\left(25{ }^{\circ} \mathrm{C}, 101 \mathrm{kPa} \mathrm{H}_{2}\right)$, 实现了对 多种烯烃的氢化(表 1), 该体系对位阻比较敏感, 取代基 较小的烯烃更易被氢化.

Grant 等 ${ }^{[12]}$ 对在紫外光照射下 $\mathrm{Fe}(\mathrm{CO})_{5}$ 催化烯烃气 相氢化反应进行了深入研究, 并给出了该反应可能的机 理(Scheme 3). 在这个机理中, 紫外光促进 $\mathrm{Fe}(\mathrm{CO})_{5}$ 的羰 基解离, 生成配位不饱和的 $\mathrm{Fe}(\mathrm{CO})_{3}$, 该中间体能够分 别活化氢气和烯烃, 通过插入和还原消除等步骤实现烯 烃的氢化.

\section{2 含氮配体的零价铁络合物催化剂}

1998 年, Brookhart 等 ${ }^{[13]}$ 发展了一类吡啶双亚胺配 体和铁的络合物 6 , 并发现该络合物能够高效地催化烯 烃的聚合反应. 2004 年, Chirik 等 ${ }^{[14]}$ 将络合物 6 在氮气氛 围下, 用钠采齐或 $\mathrm{NaBEt}_{3} \mathrm{H}$ 还原得到零价铁络合物 7 (Scheme 4). 后来, 他们通过穆斯堡尔谱、IR, NMR 以
表 1 紫外线照射下 $\mathrm{Fe}(\mathrm{CO})_{5}$ 催化的烯烃氢化反应

Table 1 Pentacarbonyliron(0)-catalyzed hydrogenation of olefins under ultraviolet irradiation

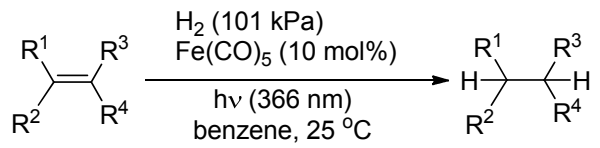

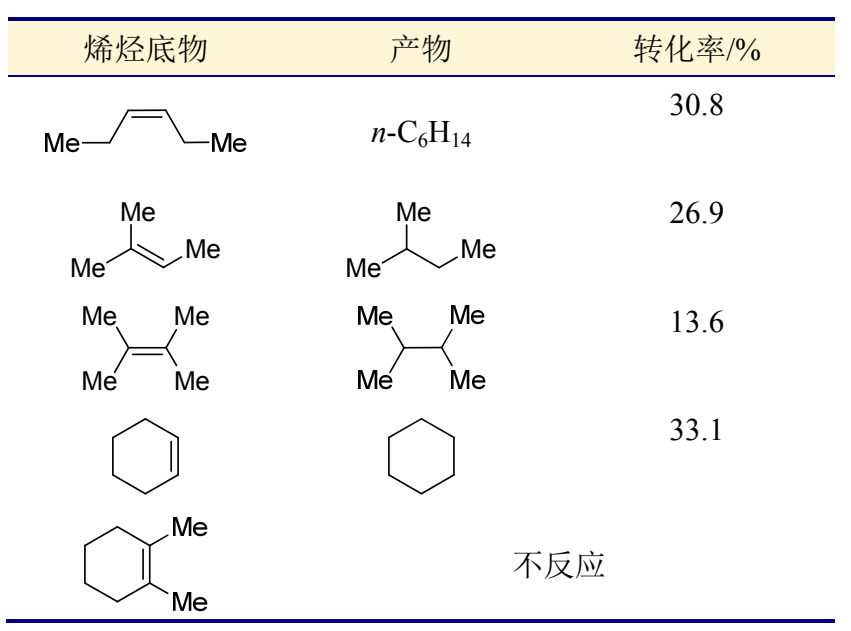

及理论计算(DFT)对络合物 7 的结构进行了详细研究, 发现吡啶双亚胺配体能够逐步从铁上获得电子, 形成新 颖的自由基结构 $7^{\prime}$ 和 $7^{\prime{ }^{[15]}}$.

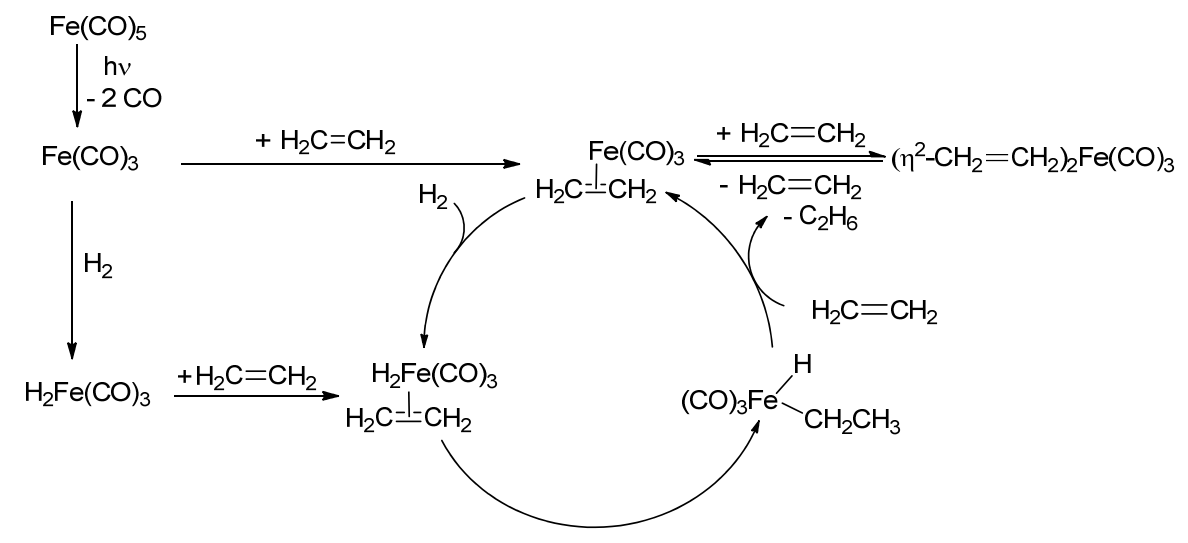

图式 $3 \mathrm{Fe}(\mathrm{CO})_{5}$ 催化烯烃氢化反应的可能机理

Scheme 3 Proposed mechanism of pentacarbonyliron(0)-catalyzed hydrogenation of olefins

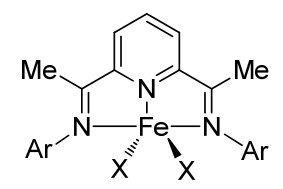

$$
\mathrm{Ar}=2,6-(i-\operatorname{Pr})_{2}-\mathrm{C}_{6} \mathrm{H}_{3}
$$

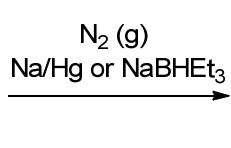

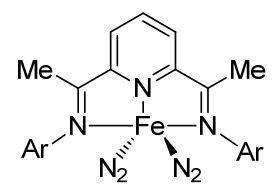

6a $(X=C l), 6 b(X=B r)$

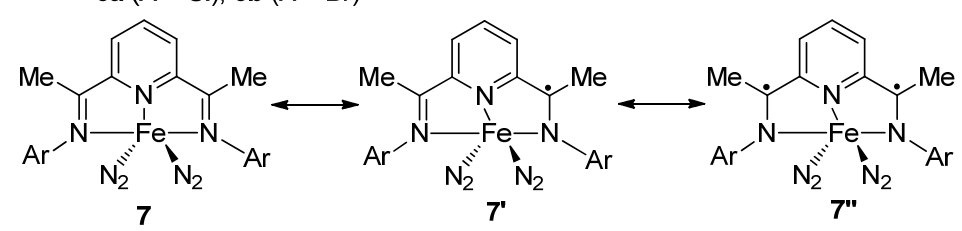

图式 4 吡啶双亚胺配体与铁的络合物

Scheme 4 Iron complexes of pyridine-diimine ligands 
络合物 7 能够在温和的反应条件下(室温, $405 \mathrm{kPa}$ $\mathrm{H}_{2}$ ) 高效地催化多种烯烃的氢化反应(表 2). 催化剂 7 对端 烯和二取代烯烃表现出了很高的活性, TON 最高达 2500, TOF 最高达 $1814 \mathrm{~h}^{-1}$ (Entries 1 5); 对醚基、酯基、取代 氨基有比较好的耐受性(Entries 6 9), 但是对含有自由 氨基、酰胺基和螯合性双酯基取代的烯烃催化活性较低 (Entries $10 \sim 12$ ), 而对于烯丙酯和环己烯酮基本没有催 化活性(Entries 13，14), 其中环己烯酮造成了催化剂的失 活.

表 2 络合物 7 催化的烯烃氢化反应

Table 2 Hydrogenation of olefins catalyzed by complexes 7

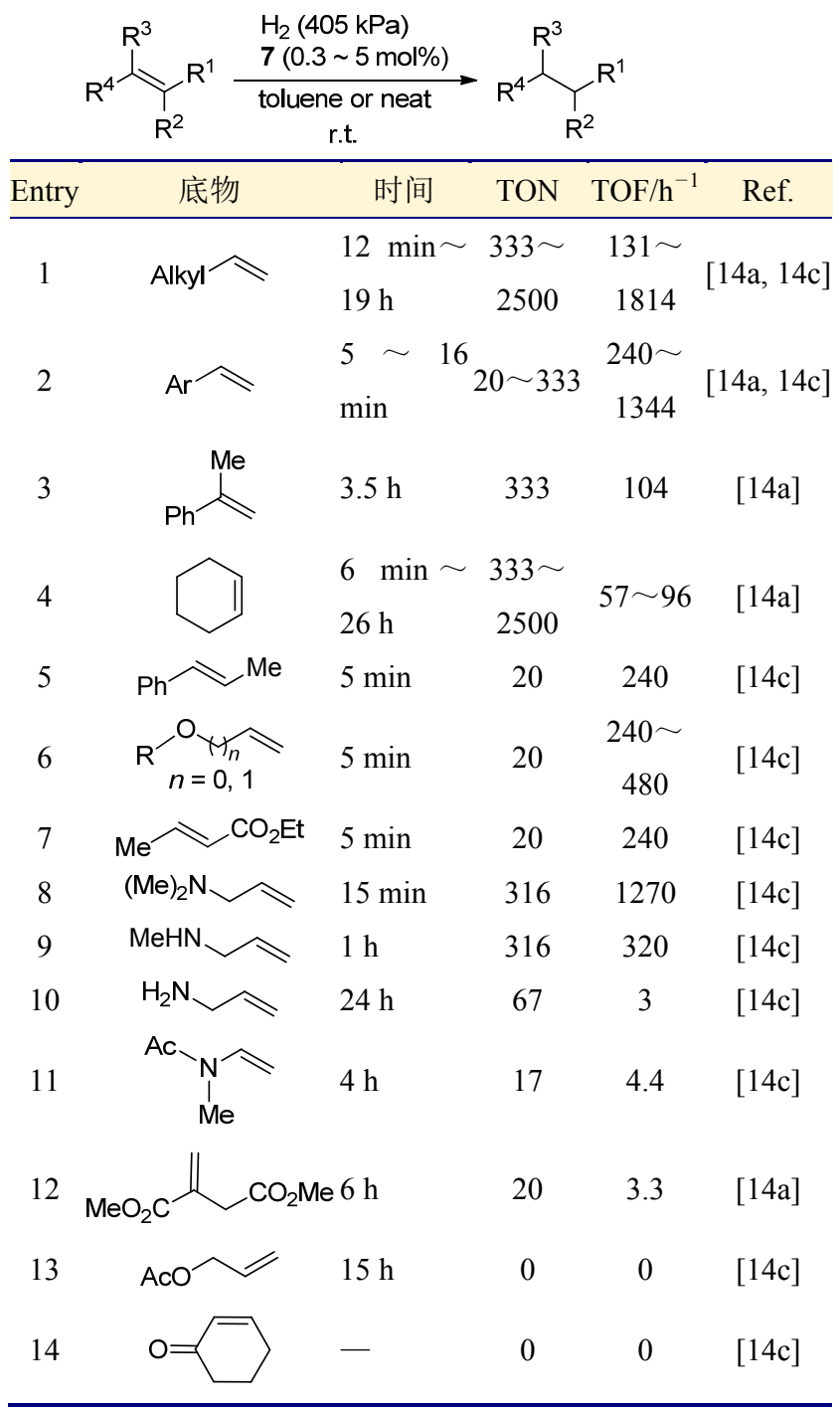

络合物 7 也可以实现内炔(如二苯基乙炔和 2-丁炔) 的氢化, 首先氢化到顺式烯烃, 然后再进一步氢化为饱 和烷烃(Scheme 5) ${ }^{[14 a]}$. 但该络合物不能催化端炔(如三 甲基硅乙炔)的氢化.

值得一提的是，在相同的反应条件下，络合物 7 的催 化烯烃氢化的活性甚至比经典的非均相催化剂 $(\mathrm{Pd} / \mathrm{C})$ 和 均相催化剂(Wilkinson 催化剂和 Crabtree 催化剂)还要高

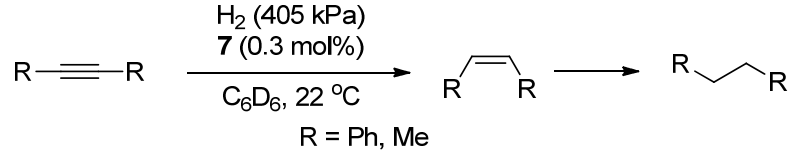

图式 5 络合物 7 催化内炔的氢化反应

Scheme 5 Hydrogenation of internal alkynes catalyzed by complex 7

(表 3), 表明了铁催化剂在烯烃氢化反应中的巨大潜力.

表 3 络合物 7 与其它过渡金属催化剂的活性比较

Table 3 Comparison of catalyst 7 and other known hydrogenation catalysts

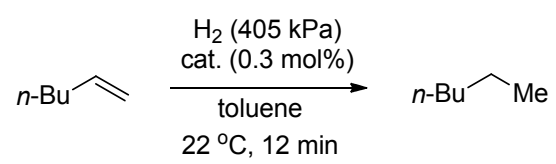

\begin{tabular}{|c|c|}
\hline Cat. & $\mathrm{TOF} / \mathrm{h}^{-1}$ \\
\hline 7 & 1814 \\
\hline $10 \% \mathrm{Pd} / \mathrm{C}$ & 366 \\
\hline $\mathrm{RhCl}\left(\mathrm{PPh}_{3}\right)_{3}($ Wilkinson catalyst $)$ & 10 \\
\hline$\left[\operatorname{Ir}(\mathrm{COD})\left(\mathrm{PCy}_{3}\right) \mathrm{Py}\right] \mathrm{PF}_{6}(\text { Crabtree catalyst })^{a}$ & 75 \\
\hline
\end{tabular}

${ }^{a} \mathrm{COD}=1,4$-环辛二烯; $\mathrm{Py}=$ 吡啶; $\mathrm{PCy}_{3}=$ 三环己基膦.

Chirik 等 ${ }^{[14 a]}$ 提出了该氢化反应的可能机理(Scheme 6). 零价铁络合物 7 首先解离两个氮分子, 并与烯烃配 位，然后通过氧化加成的方式活化 $\mathrm{H}_{2}$ ，产生二价铁一负

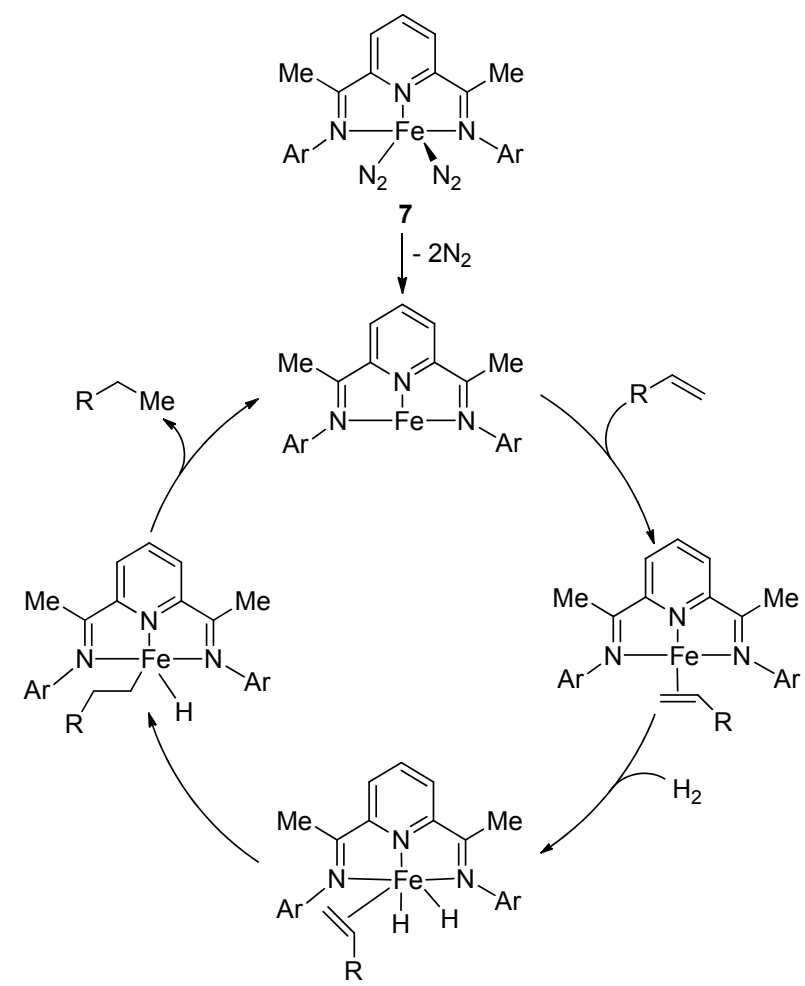

图式 6 络合物 7 催化烯烃氢化的可能历程

Scheme 6 Proposed mechanism of the hydrogenation of olefins catalyzed by complex 7 
氢中间体，再经历烯烃的插入和还原消除等后续步骤， 完成催化循环.

Chirik 等 ${ }^{[16]}$ 用制备络合物 7 的类似方法，合成了一种 新的吡定双亚胺配体的铁络合物 $\mathbf{8}$, 双亚胺类零价铁络 合物 $9^{[17]}$ 和含有钳式膦-氮配体的铁负氢络合物 $\mathbf{1 0}^{[18]}$. 络 合物 8 在 1-已烯的氢化反应中表现出比 7 更高的催化活 性, 但是在多取代烯烃的氢化中活性较低. 络合物 9 和 10 在烯烃氢化中的活性明显低于络合物 7 (Eq. 1).

$$
\frac{\text { cat. }(0.3 \mathrm{~mol} \%)}{\text { pentane, } 22^{\circ} \mathrm{C}}
$$

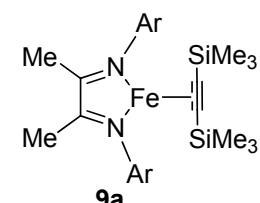

$9 a^{A}$<smiles></smiles>
$9 b$

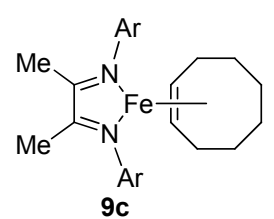

TOF $=90 \mathrm{~h}^{-1}$
TOF $=4 \mathrm{~h}^{-1}\left(\right.$ at $\left.60^{\circ} \mathrm{C}\right) \quad$ TOF $=90 \mathrm{~h}^{-1}$

2012 年, Chirik 等 ${ }^{[19]}$ 又对催化剂 7 进行了改造, 以 $N$-杂环卡宾替代亚胺结构, 发展了新一类催化烯烃氢化 的络合物 11. 与吡啶双亚胺的铁络合物 7 相比, 络合物 11 在三取代或四取代环状烯烃的氢化反应中表现出更 高的活性(表 4). 但是目前这两类铁络合物催化剂都无 法实现四取代链状烯烃的氢化反应.

\section{3 多齿膦配体的铁络合物催化剂}

1989 年, Bianchini 等 ${ }^{[20]}$ 以四齿膦配体和铁的络合物 12 作为催化剂前体, 实现了对端炔的氢化(Eq. 2). 该反 应能够停留到烯烃阶段, 即使延长反应时间, 也只能生 成极少量完全氢化的烷烃产物. 络合物 12 比较稳定, 反 应后可以回收使用。

$$
\begin{aligned}
& \mathrm{R} \rightleftharpoons \frac{\mathrm{H}_{2}(101 \mathrm{kPa})}{\mathrm{THF}(1 \mathrm{~mol} \%)} \underset{\mathrm{TOF}=21.2 \sim 45.2 \mathrm{~h}^{\circ} \mathrm{C}}{\longrightarrow} \\
& \mathrm{R}=\mathrm{Ph}, n-\mathrm{C}_{3} \mathrm{H}_{7}, n-\mathrm{C}_{5} \mathrm{H}_{11},- \text { 多 } \mathrm{OMe} \\
& { }_{12}
\end{aligned}
$$


位(步骤 b), 然后催化剂上的负氢与炔烃发生插入反应, 生成含铁-碳键的中间体(步骤 $\mathrm{c}$ ), 该中间体与氢气作用, 发生氢解, 完成氢化反应(步骤 $\mathrm{d}, \mathrm{e}$ ). 在此催化循环中铁 的价态保持不变(始终为 +2 价).

2004 年, Peters 和 Daida ${ }^{[21]}$ 报道了一类含硼的三齿膦 配体和铁的络合物 13 催化的烯烃和炔烃的氢化反应, 虽然反应条件比较温和, 但是活性通常不高(Eq. 3).

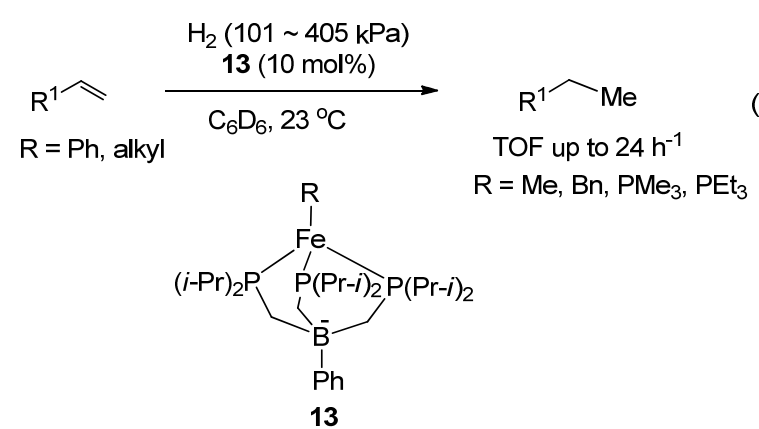

2013 年, Peters 等 ${ }^{[22]}$ 在上述工作的基础上, 使用苯 环代替 13 中的亚甲基侧链, 发展了另一类刚性更强的 含硼三齿膦配体的零价铁络合物 $\mathbf{1 4}$ (Eq. 4). 络合物 $\mathbf{1 4}$ 能实现对端烯和端炔的氢化反应，其反应性和 13 类似.

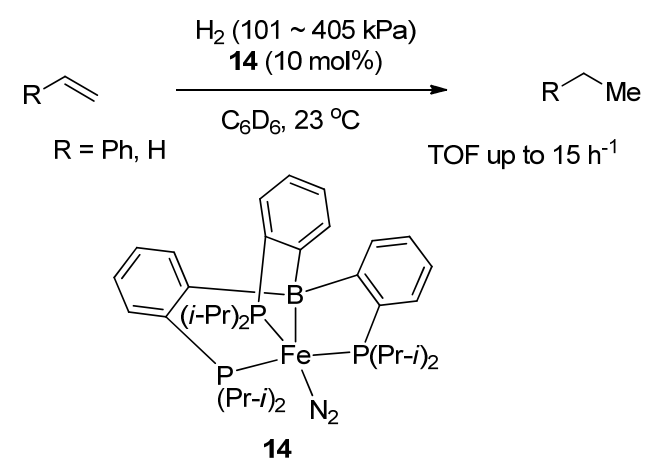

机理研究表明, 铁硼化合物 14 中铁硼键的形成和 断裂是可逆的, 这一过程能够促进对氢气分子的异裂, 形成含有一个氢桥键的关键中间体 15 , 进而实现对烯 烃和炔烃的氢化(Scheme 8).

Milstein 等 ${ }^{[23]}$ 发展了一类二氢呋啶骨架的钳式配体 和铁的络合物 16 催化剂, 实现了炔烃的氢化反应(Eq. $5)$. 该反应可以停留到烯烃阶段, 表现出很高的 $E$ 式选 择性. 研究表明, 催化剂 16 可以促进 $Z$ 式烯烃向 $E$ 式烯 烃的转变, 这是反应表现出 $E$ 式选择性的根本原因.

\section{4 原位生成的活性铁络合物催化剂}

虽然结构确定的铁络合物催化剂对机理研究很有 帮助, 但是这些络合物往往难以制备, 稳定性较差, 在 实际使用中有很多困难. 因此人们又发展了一些原位制 备铁催化剂的方法.

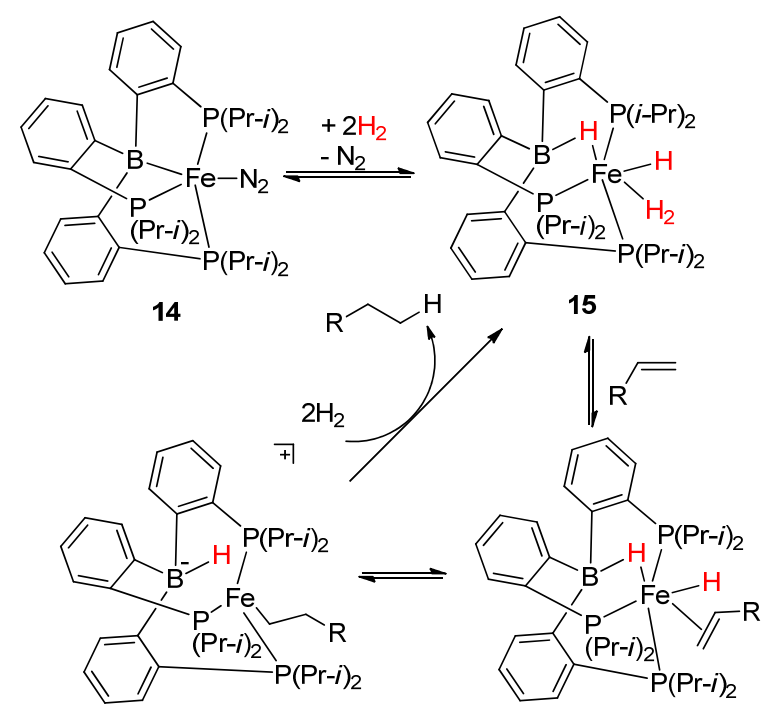

图式 8 络合物 14 催化烯烃氢化反应的可能机理

Scheme 8 Proposed mechanism of the hydrogenation of olefins catalyzed by complex 14

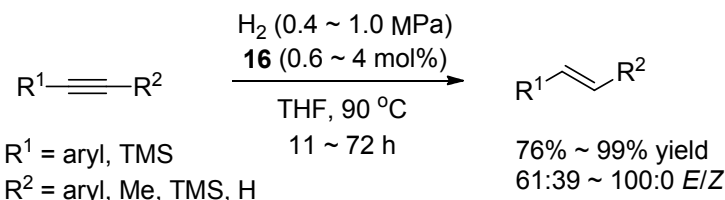

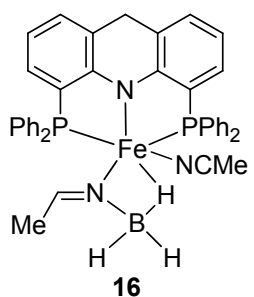

1968 年, Tajima 和 Kunioka ${ }^{[24]}$ 以 $\mathrm{AlEt}_{3}$ 和 $\mathrm{PhMgBr}$ 作为活化试剂, 实现了 $\left[\mathrm{CpFe}(\mathrm{CO})_{2} \mathrm{Cl}\right]$ 催化共轭烯烃(比 如 1,3-丁二烯)的氢化, 产品主要是 cis-和 trans-2-丁烯 (接近 $1: 1$ 的混合物), 同时还观察到少量的 1-丁烯的产 生. 但是催化剂对单烯烃是没有活性的, 因为最终产品 中未见正丁烷的生成(Eq. 6).

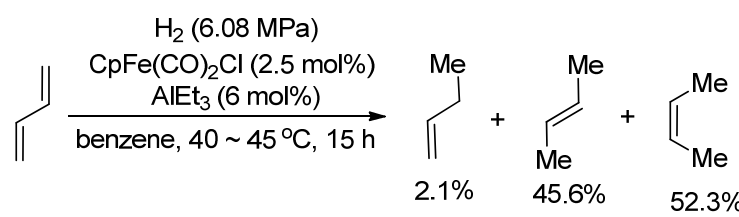

研究认为该氢化反应包含以下四步(Scheme 9): (i) 铁络合物和金属铝发生配体交换，生成烷基铁中间体; (ii) 烷基铁中间体发生氢解生成烷烃和铁-负氢中间体; (iii)烯烃对铁一负氢键进行插入, 生成新的烷基铁化合 物; (iv)最后再次和氢气发生氢解反应, 生成氢化产物, 完成催化循环. 


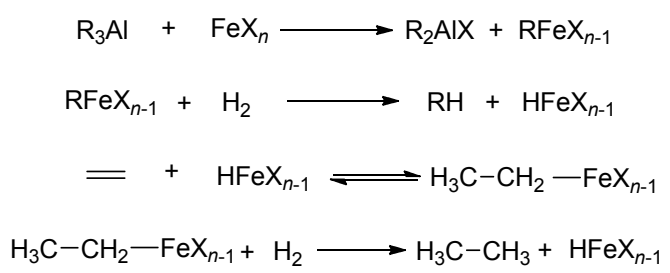

图式 $9 \mathrm{CpFe}(\mathrm{CO})_{2} \mathrm{Cl} / \mathrm{AlEt}_{3}$ 催化氢化反应的可能机理

Scheme 9 Proposed mechanism of the hydrogenation of olefins catalyzed by $\mathrm{CpFe}(\mathrm{CO})_{2} \mathrm{Cl} / \mathrm{AlEt}_{3}$

Inoue 等 ${ }^{[25]}$ 模拟氢化酶的活性位点, 使用硫铁簇合 物 17 作为催化剂前体, 在苯基锂的活化下, 实现了烯烃 的氢化(Eq. 7). 该反应中, 苯基锂的用量对催化剂的活 性有明显的影响, 当苯基锂的用量为硫铁簇合物的 8 倍 时催化效果最好. 活性催化剂的生成模式应该和 Scheme 9 所示的类似.

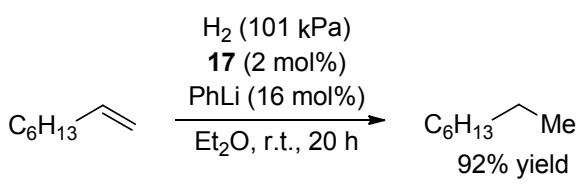

$$
\begin{aligned}
& {\left[\begin{array}{c}
\mathrm{Cl}-\mathrm{Fe}^{\prime} \vdots-\mathrm{S}^{\prime} \mid \\
\mathrm{Cl}-\mathrm{Fe} \mid-\mathrm{S} \\
\mathrm{S}-\mathrm{Fe} \\
\mathrm{Cl} \\
17
\end{array}\right]\left[\mathrm{N}(n-\mathrm{Bu})_{4}\right]_{2}}
\end{aligned}
$$

最近, Thomas 等 ${ }^{[26]}$ 用格氏试剂原位还原稳定的二 价铁络合物生成活性铁催化剂, 实现了对多种一取代和 二取代烯烃的氢化, TON 最高达 33 , TOF 最高达 $33 \mathrm{~h}^{-1}$ (Eq. 8).
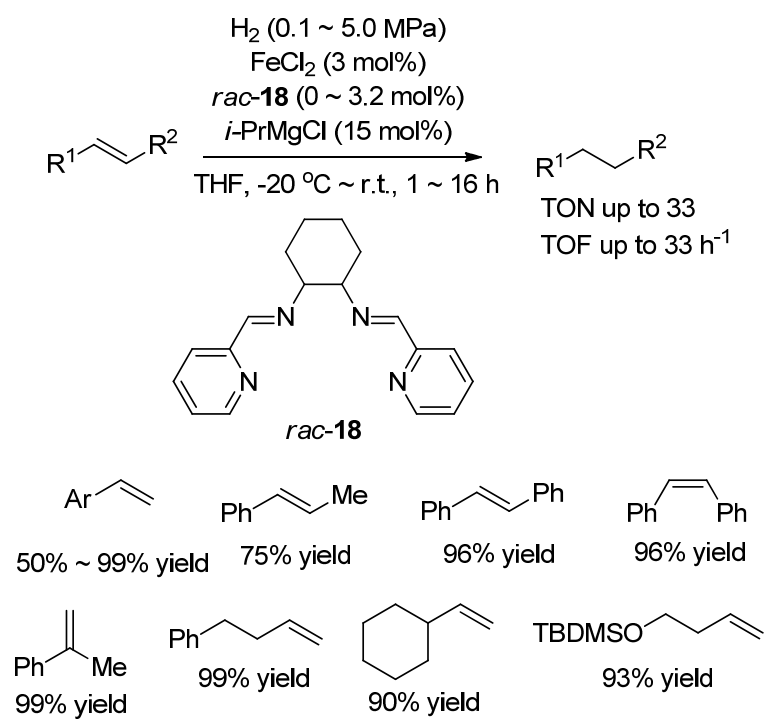

\section{5 非均相铁催化烯烃的氢化反应}

和贵金属一样, 铁纳米颗粒(FeNPs)也可以催化烯 烃和炔烃的氢化. 这类研究的关键是发展合成铁纳米颗
粒的方法. 2009 年, de Vries 等 ${ }^{[27]}$ 利用 $\mathrm{EtMgCl}$ 在乙梄或 THF 中还原 $\mathrm{FeCl}_{3}$ 得到可溶性 $\mathrm{Fe}-\mathrm{NPs}$, 平均直径为 $(2.67 \pm 0.60) \mathrm{nm}$. 该 Fe-NPs 能够在温和条件下 $(0.1 \mathrm{MPa}$ $\mathrm{H}_{2}$, 室温)催化一取代和二取代烯烃以及炔烃的氢化反 应, 表现出较高的活性(Eq. 9). 但是该 Fe-NPs 催化剂对 底物的位阻及官能团都很敏感，无法氢化三取代或四取 代烯烃, 也不能催化不饱和羧酸酯、 $\alpha$-脱氢氨基酸等官 能团烯烃的氢化. 对于炔烃的催化氢化, 很难停留在烯 烃阶段, 而主要得到完全氢化的烷烃产物.

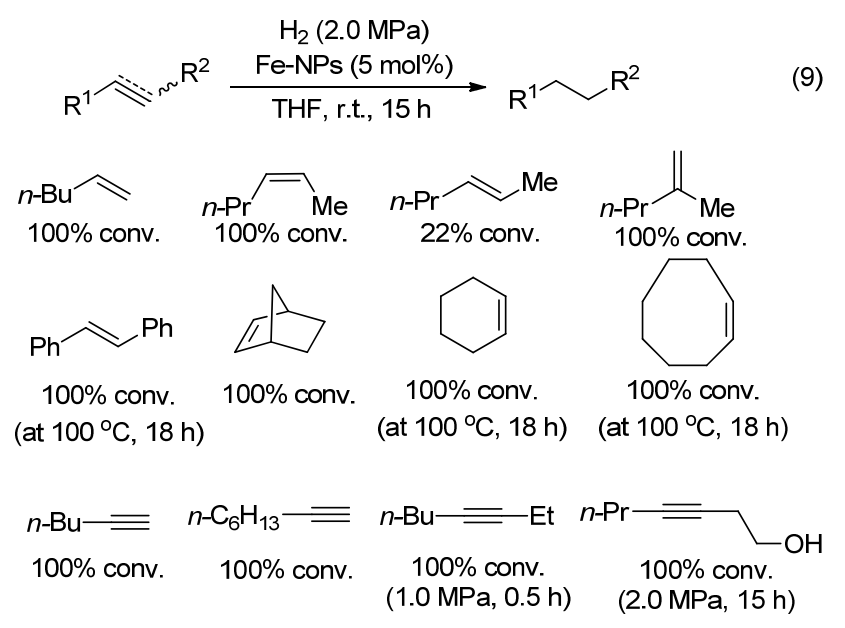

Lefort 和 de Vries 等 ${ }^{[28]}$ 通过动力学研究, 提出了 Fe-NPs 催化炔烃氢化的可能机理(Scheme 10). 该氢化 反应的关键是活性的铁表面分解氢气产生铁一负氢中间 体, 然后经由炔烃的插入，氢解等步骤完成氢化，得到 烯烃或烷烃产物.

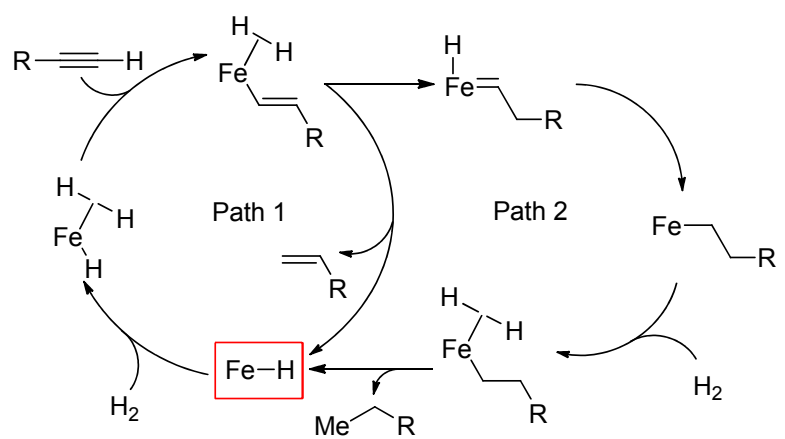

图式 10 铁纳米颗粒催化烯烃和炔烃氢化反应的可能机理 Scheme 10 Proposed mechanism of the hydrogenation of olefins and alkynes catalyzed by Fe-NPs

Bauer 和 von Wangelin 等 ${ }^{[29]}$ 采用相似的方法, 用 $\mathrm{EtMgCl}$ 还原 $\mathrm{FeCl}_{3}$ 得到了 $\mathrm{THF}$ 稳定的 $\mathrm{Fe}(0)$ 族(直径约为 $5 \AA$ ), 并用该簇合物催化了烯丙苯、苯乙烯等的氢化(Eq. 10).

随后, Prechtl 和 von Wangelin 等 ${ }^{[30]}$ 又利用 $\mathrm{EtMgCl}$ 还原 $\mathrm{FeCl}_{3}$ 得到铁纳米颗粒 $\mathrm{FeNPs}$ (约 $5 \mathrm{~nm}$ ), 在正己烷/ 


$$
\begin{aligned}
& \mathrm{H}_{2}(0.4 \text { or } 3.0 \mathrm{MPa}) \\
& \mathrm{FeCl}_{3}(5 \mathrm{~mol} \%) \\
& \mathrm{R}^{1} \leftrightharpoons \mathrm{R}^{2} \stackrel{\mathrm{EtMgCl}(10 \sim 30 \mathrm{~mol} \%)}{\mathrm{THF}, 20 \text { or } 45^{\circ} \mathrm{C}} \rightarrow \mathrm{R}^{1} \mathrm{R}^{2} \\
& \mathrm{R}^{1}=\text { aryl, benzyl } \quad 3 \sim 16 \mathrm{~h} \quad 36 \% \sim 99 \% \text { yield } \\
& \mathrm{R}^{2}=\mathrm{H}, \mathrm{Ph}
\end{aligned}
$$

离子液体(IL)的两相体系中实现了内炔的氢化反应(Eq. 11). 在 IL 上引入氧基或在体系中加入乙腈, 可以控制 反应停在烯烃阶段, 得到 $Z$ 式烯烃. IL 的使用不仅有利 于 Fe-NPs 的溶解和稳定, 还有利于催化剂的分离以及 循环使用.

$$
\begin{aligned}
& \mathrm{H}_{2}(6.0 \mathrm{MPa}) \\
& \mathrm{FeCl}_{3}(5 \mathrm{~mol} \%) \\
& \mathrm{EtMgCl}(20 \mathrm{~mol} \%)
\end{aligned}
$$

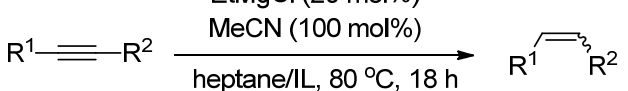

$$
\begin{aligned}
& \begin{array}{ll}
\mathrm{R}^{1}=\text { aryl, alkyl } & 13 \% \sim 98 \% \text { yield } \\
\mathrm{R}^{2}=\text { aryl, alkyl, TMS, } \mathrm{CO}_{2} \mathrm{Me} & 92: 8 \sim 100: 0 \text { Z/E }
\end{array} \\
& \mathrm{IL}=\mathrm{Me}_{-\mathrm{NTf}}^{\mathrm{N}=} \mathrm{Me}_{2}^{\mathrm{N}-\mathrm{Bu}-n}
\end{aligned}
$$

Breit 等 ${ }^{[31}$ 通过超声波分解 $\mathrm{Fe}(\mathrm{CO})_{5}$ 得到负载在化学 修饰的石墨烯(CDG)上的 FeNPs, 负载率为 $3.66 \mathrm{wt} \%$, 颗粒平均直径为 $(4.37 \pm 1.62) \mathrm{nm}$, 且具有很高的比表面 积. 该负载型纳米铁颗粒能够有效催化端烯、环状烯烃 和二取代炔烃的氢化, 反应温度为 $100{ }^{\circ} \mathrm{C}$, 氢气压力为 $2 \mathrm{MPa}, \mathrm{TON}$ 最高达 111 , 但对链状二取代或多取代烯烃 和端炔几乎没有活性(Eq. 12). 该 Fe-NP/CDG 需要加入 格氏试剂活化, 消除氧化膜, 才能得到活性更高的铁催 化剂. 反应结束后, $\mathrm{Fe}-\mathrm{NP} / \mathrm{CDG}$ 可以由简单的磁倾析分 离，并且能循环利用多次而不丧失活性.

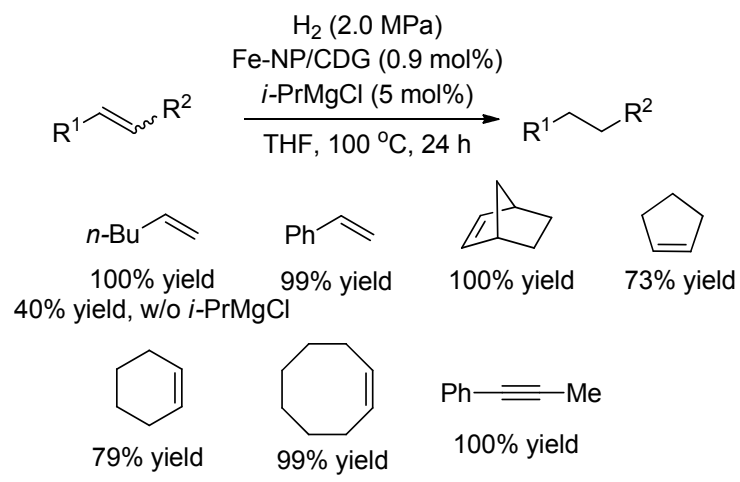

Chaudret等 ${ }^{[32]}$ 将 $\left\{\mathrm{Fe}\left(\mathrm{N}\left[\mathrm{Si}\left(\mathrm{CH}_{3}\right)_{3}\right]_{2}\right)_{2}\right\}_{2}$ 在 $\mathrm{H}_{2}$ 氛围加热 至 $150{ }^{\circ} \mathrm{C}$ 使其降解, 得到超小 Fe-NPs $(1.5 \pm 0.2 \mathrm{~nm})$. 该 $\mathrm{Fe}-\mathrm{NPs}$ 在室温和氢气压力为 $1.0 \mathrm{MPa}$ 条件下就能够有效 催化多种一取代和二取代烯烃和炔烃的氢化(Eq. 13),
并且循环使用 3 次活性仍然保持.

$$
\begin{aligned}
& \mathrm{H}_{2} \text { (1.0 MPa) }
\end{aligned}
$$

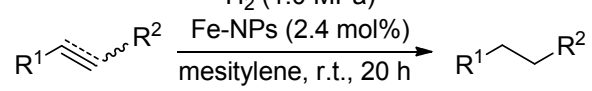

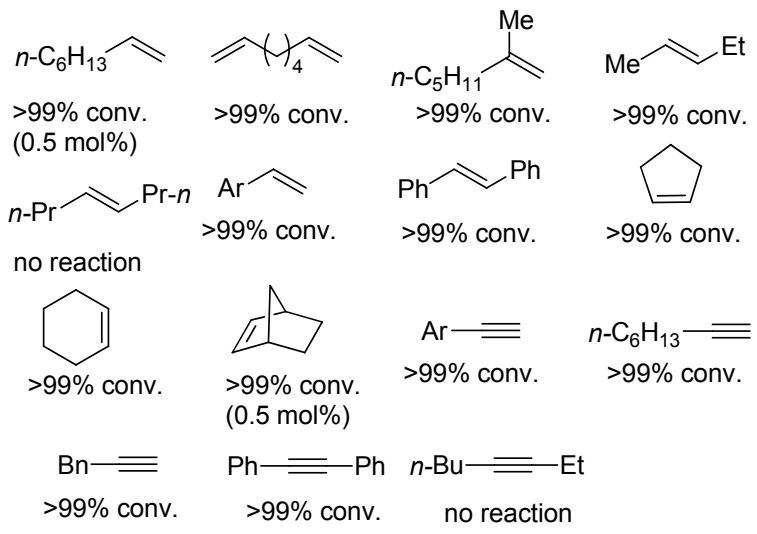

Moores 等 ${ }^{[33]}$ 用 $\mathrm{NaBH}_{4}$ 在水/甲醇的混合溶液中还原 $\mathrm{FeSO}_{4}$ 得到一种具有壳层结构的氧化铁包裹的纳米铁颗 粒 $(\mathrm{Fe}-\mathrm{CSNPs})$, 其平均核直径为 $(44 \pm 8.3) \mathrm{nm}$, 壳层厚 度为 $(6 \pm 2) \mathrm{nm}$. 该 Fe-CSNPs 可以在乙醇中催化烯烃或 炔烃氢化反应, 对一取代端烯、具有较大环张力的降冰 片烯以及端炔都有较高的活性，对羰基没有活性(Eq. 14). 由于独特的壳层结构, Fe-CSNPs 对水和氧气表现 了一定的耐受性，当在体系中加入少量水时，不影响反 应结果, 并可以方便地通过磁倾析分离, 重复使用 10 次 仍能保持较高的活性.

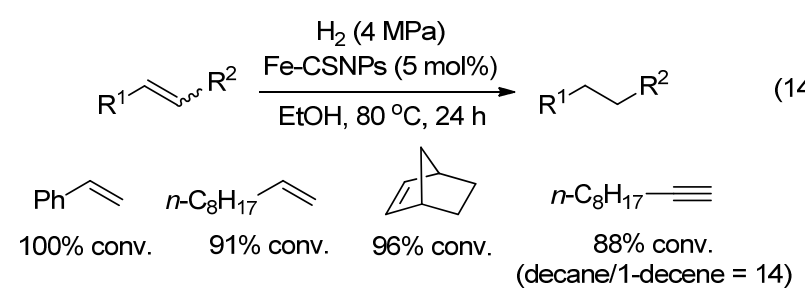

\section{2 醛、酮和亚胺的催化氢化}

过渡金属催化醛、酮和亚胺的氢化是合成醇和胺的 有效方法. 和简单烯烃相比，醛、酮和亚胺的双键具有 明显的极性，其氢化过程通常包含一步金属负氢对该极 性键的加成，随后发生质子解得到氢化产物. 由于基于 钓的双功能催化体系可以通过六元环过渡态高效地实 现对醛、酮、亚胺的氢化反应(Eq. 15)，人们受此启发，发 展出了一系列双官能的铁催化剂, 实现了这些极性双键 的氢化反应 ${ }^{[34]}$.

\section{1 半夹心羰基铁络合物催化剂}

虽然早在 1999 年, Knölker 等 ${ }^{[35]}$ 就合成了与 Shov 催 化剂 ${ }^{[36]}$ 具有明显类似性的半夹心结构羰基铁络合物 19 , 
<smiles>CC(=O)c1ccccc1</smiles>

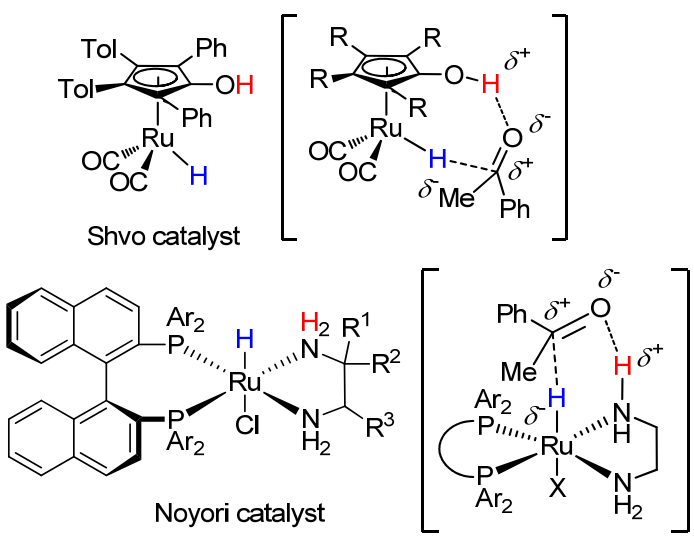

但是直到 2007 年, Casey 和 Guan ${ }^{[37]}$ 才首次将络合物 19 用于酮的氢化反应中. 在温和的反应条件下，19 能够催 化多种醛、酮的氢化反应, 得到中等的 TON 和 TOF (Eq. 16). 该催化剂对醛、酮有较好的选择性, 它不能氢化环 氧、苯甲酸酯、炔烃和烯烃, 因此对这些官能团有较好 的耐受性. 由于转移氢化和氢化反应具有相似的机理, 该催化剂也能以异丙醇为氢源实现酮的转移氢化反应.
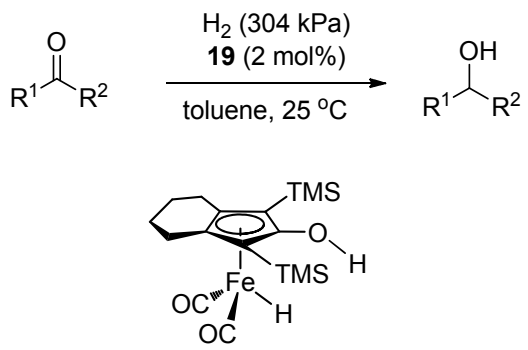

19

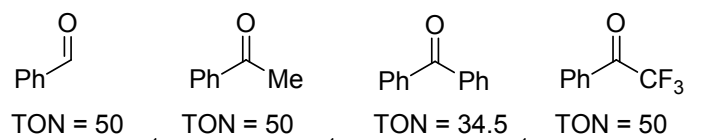

TOF $=25 \mathrm{~h}^{-1} \quad$ TOF $=2.5 \mathrm{~h}^{-1} \quad$ TOF $=0.5 \mathrm{~h}^{-1} \quad$ TOF $=300 \mathrm{~h}^{-1}$<smiles>CC(=O)CCc1ccccc1</smiles><smiles>C=CCCC(C)=O</smiles>

催化剂 19 还可以实现 $\alpha, \beta$-不饱和酮的氢化, 但是得 到的是羰基被氢化的产物烯丙醇和完全氢化的产物饱 和醇(Eq. 17). 在相同条件下, 该催化剂也能实现醛亚胺 的氢化, 但是活性稍低, 需要升温到 $65{ }^{\circ} \mathrm{C}$ 下反应( Eq.
18).<smiles>CC(=O)/C=C/c1ccccc1</smiles>

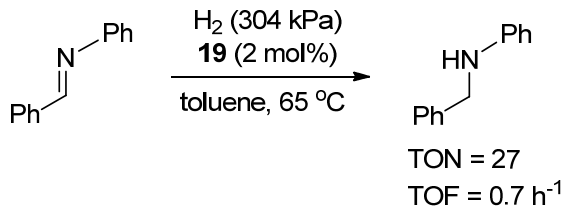

Casey 和 Guan ${ }^{[38]}$ 分离到醇和铁催化剂的中性络合 物 22 的单晶, 通过研究络合物 22 与醇的配体交换反应, 建议该反应可能经由球外协同的机理(即羰基未和铁配 位)完成的(Scheme 11). 催化剂 19 通过协同的历程, 经 由过渡态 20, 同时向羰基转移负氢和质子, 得到具有空 轨道的中间体 21, 然后和醇进行配位, 得到在低温下可 以分离的中性铁络合物 22. 中间体 21 通过空轨道和氢 气配位 $(21 \sim 23)$, 在产物醇的协同下实现氢气的异裂 $(23,24)$, 重新生成催化剂 19 , 完成催化循环.

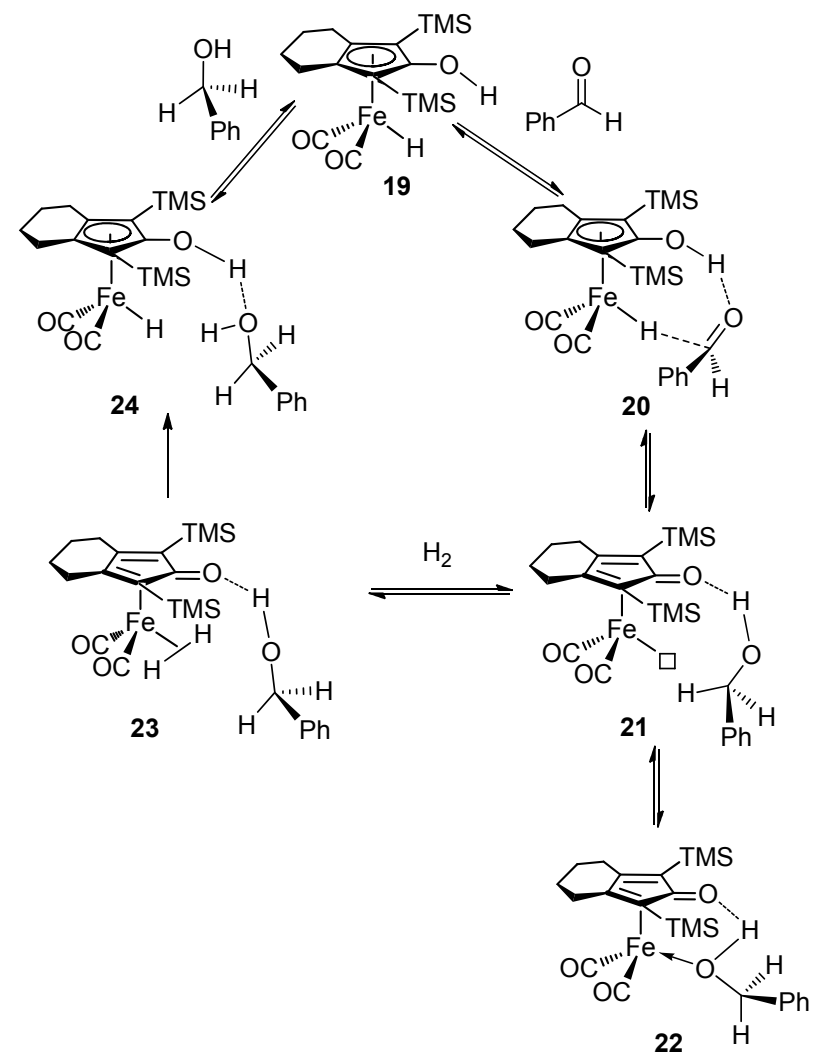

图式 11 铁络合物 19 催化㨏基化合物氢化反应的可能机理 Scheme 11 Proposed mechanism of the hydrogenation of carbonyl compounds catalyzed by iron complex 19 
Chen ${ }^{[39]}$ 和 Zhang 等 ${ }^{[40]}$ 分别通过密度泛函(DFT)计算 的方法对该反应的机理进行了研究, 发现协同的六元环 过渡态 20 从动力学和热力学上都是有利的.

由于铁络合物 19 稳定性较差, 因此人们尝试原位 制备该活性催化剂用于氢化反应. Renaud 等 ${ }^{[41]}$ 发展了一 种由稳定的羰基铁络合物 25 合成活性铁络合物 19 的方 法(Scheme 12). 他们用三甲胺的氮氧化物作为氧化剂, 将化合物 25 的一个羰基配体氧化成二氧化碳, 生成配 位不饱和的中间体 26, 然后在氢气作用下, 生成活性催 化剂 19. 络合物 25 被成功应用于醛或酮的还原氨化反 应(Scheme 13). 在该反应中, 醛或酮与胺现场生成亚 胺, 然后同样经过球外协同的机理(中间体 27)被氢化, 得到伯胺或者季胺化合物. 该方法避免分离较不稳定的 亚胺或烯胺中间体, 在合成上有明显优势, 其成功的关 键在于铁催化剂能够在醛、酮存在下选择性地氢化亚胺.
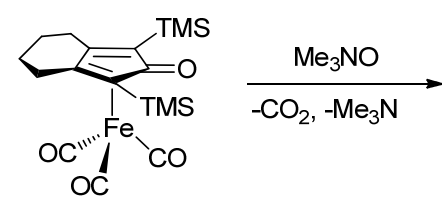

25

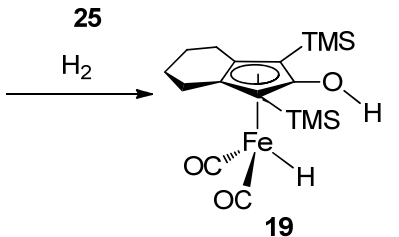

图式 12 氧化法原位生成活性催化剂 19

Scheme 12 In situ generation of 19 through oxidation of $\mathrm{Me}_{3} \mathrm{NO}$

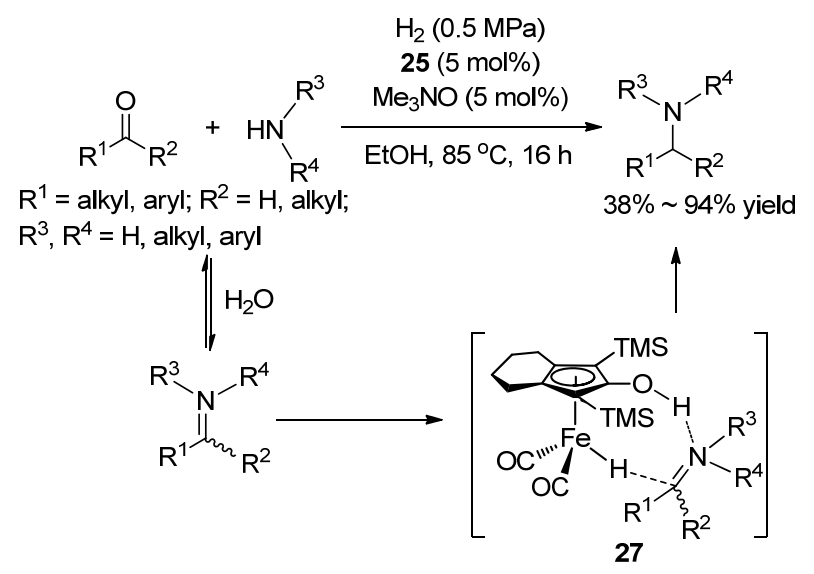

图式 13 铁络合物 25 催化的还原胺化反应

Scheme 13 Reductive amination of aldehydes and ketones catalyzed by iron complex $\mathbf{2 5}$

Renaud 等 ${ }^{[42]}$ 还在铁络合物 $\mathbf{2 8}$ 的茂环上引入季胺结 构, 提高其在水中的溶解度, 在水中实现了对醛、酮和 亚胺的氢化反应(Eq. 19).

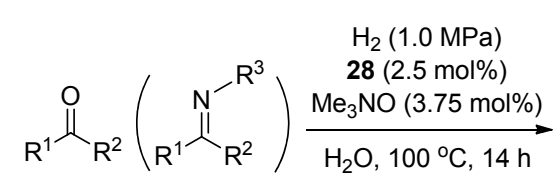

$$
\underset{18 \% \sim 98 \% \text { yield }}{\stackrel{\mathrm{OH}}{\mathrm{R}_{\mathrm{R}^{2}}}\left({ }_{\mathrm{R}^{1}}^{\mathrm{HN}_{\mathrm{R}^{2}}^{-\mathrm{R}^{3}}}\right)}
$$

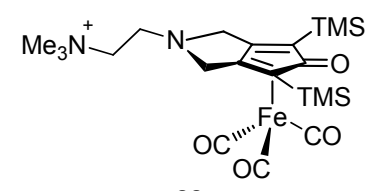

28

Beller 等 ${ }^{[33]}$ 使用络合物 $\mathbf{2 5}$ 作为催化剂前体，在催化 量碳酸钾的存在下, 实现了多种醛、酮化合物的氢化, TON 最高可达 3700 (Scheme 14). 活性催化剂生成过程 如下：在碱性条件下，氢氧根进攻络合物 $\mathbf{2 5}$ 中的一个羰 基配体, 释放出一分子二氧化碳, 生成铁负氢中间体 29, 该中间体夺取水的质子, 生成了活性催化剂 19. 但 是需要指出的是, 和最初 Casey 等用的氢化条件相比, Beller 等的氢化条件更剧烈，这有可能是反应取得更高 活性的原因之一.
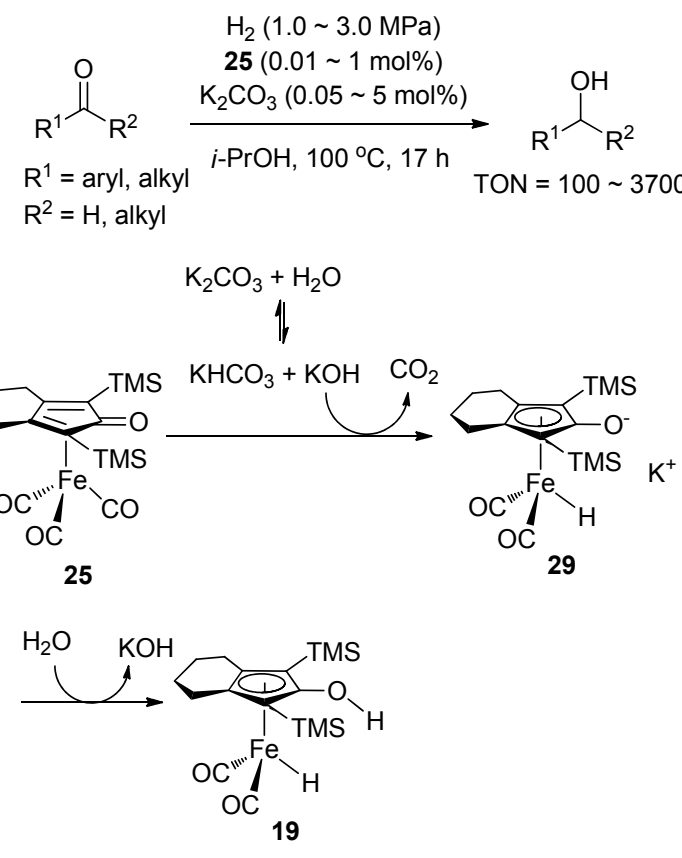

图式 14 铁络合物 25 催化醛和酮的氢化反应

Scheme 14 Hydrogenation of aldehydes and ketones catalyzed by iron complex 25

这种原位生成的催化剂可以选择性地氢化 $\alpha, \beta$-不饱 和醛的羰基而保留双键, 也表现出较高的活性(Eq. 20). 


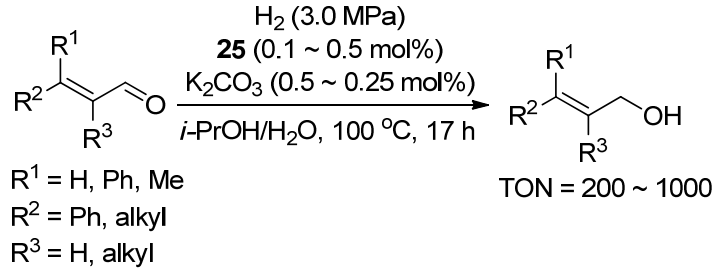

人们通过在催化剂 19 中引入手性配体或使用协同 催化的策略, 实现了对酮和亚胺的不对称催化氢化. Berkessel 等 $^{[44]}$ 在三甲胺氮氧化物存在下或光活化下, 使用手性亚磷酰胺配体 31 置换铁络合物 $\mathbf{3 0}$ 中的一个羰 基, 合成了一系列手性催化剂 32. 络合物 32 能够催化 苯乙酮的不对称氢化反应, 但是对映选择性最高只有 $32 \%$ ee (Scheme 15). 络合物 32 在氢气氛围中, 光照激 发后生成三种活性铁负氢物种 $33 \sim 35$ (Eq. 21), 其中 33 是非手性催化剂, 而 34 和 35 是一对非对映异构体. 这 种复杂的催化体系是造成反应对映选择性不高的主要 原因.

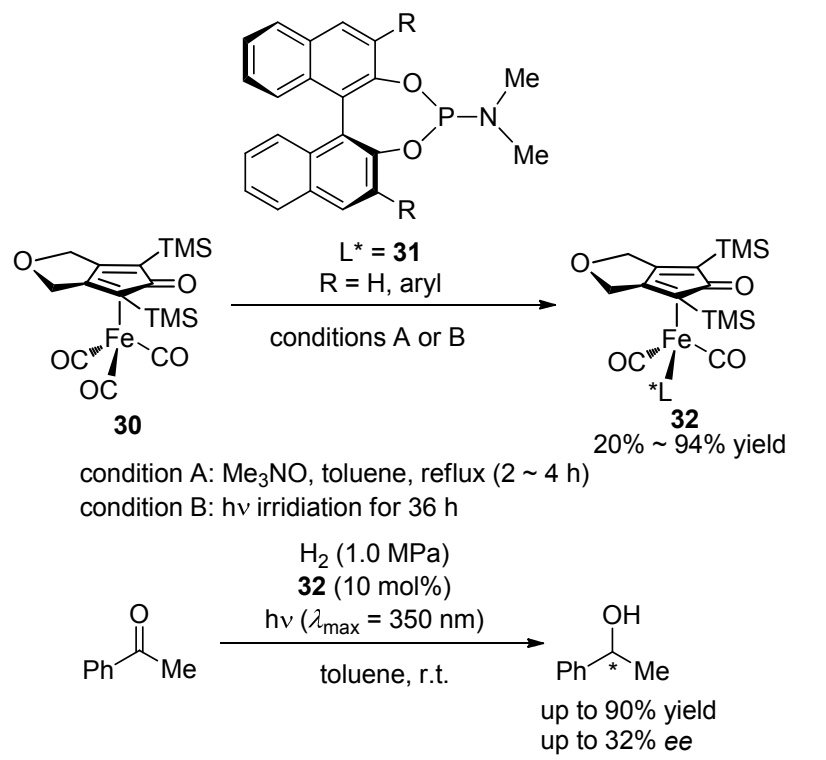

图式 15 铁络合物 32 催化苯乙酮的不对称氢化反应

Scheme 15 Asymmetric hydrogenation of acetophenone catalyzed by iron complex 32

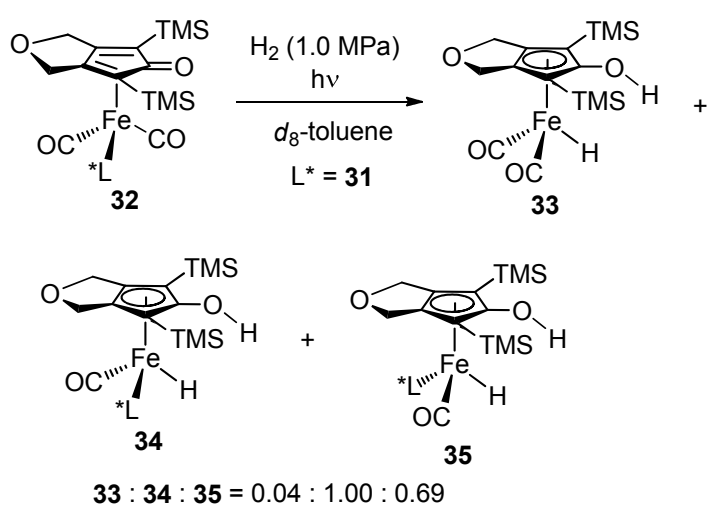

Beller 等 ${ }^{[5]}$ 运用协同催化的策略, 将催化剂 19 和手 性磷酸 $(S)-36$ 结合, 实现了酮亚胺不对称氢化反应, 取 得了很高的对映选择性(Scheme 16). 在该协同催化体系 中, 手性磷酸 $(S)-36$ 通过氢键活化亚胺, 而非手性铁络 合物 19 对活化的亚胺进行氢化. 由于手性磷酸在亚胺周 围创造了手性环境, 氢化的时候能够实现面选择性控制, 因而得到较高的对映选择性. 该体系对芳香酮亚胺非常 有效, 大多数情况下对映选择性超过 $90 \% e e$; 也能实现 多种脂肪酮亚胺的不对称氢化，对映选择性稍低.

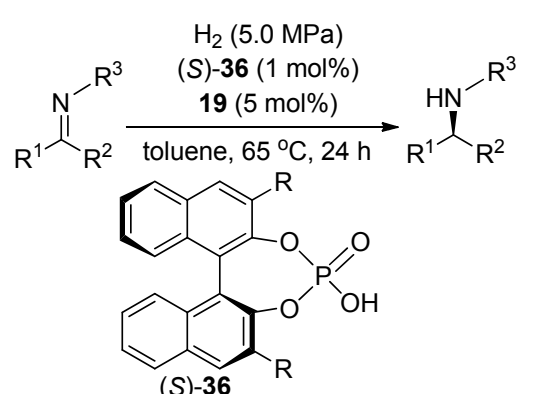

$\mathrm{R}=2,4,6-(i-\mathrm{Pr})_{3} \mathrm{C}_{6} \mathrm{H}_{2}$

$$
\begin{aligned}
\mathrm{R}^{1}= & \text { aryl, } \mathrm{R}^{2}=\text { alkyl, } \mathrm{R}^{3}=\text { aryl; } \\
& 60 \% \sim 93 \% \text { yield; } 80 \% \sim 98 \% \text { ee } \\
\mathrm{R}^{1}= & \text { alkyl or alkenyl, } \mathrm{R}^{2}=\mathrm{Me}, \mathrm{R}^{3}=\text { aryl; } \\
& 69 \% \sim 94 \% \text { yield; } 67 \% \sim 83 \% \text { ee }
\end{aligned}
$$

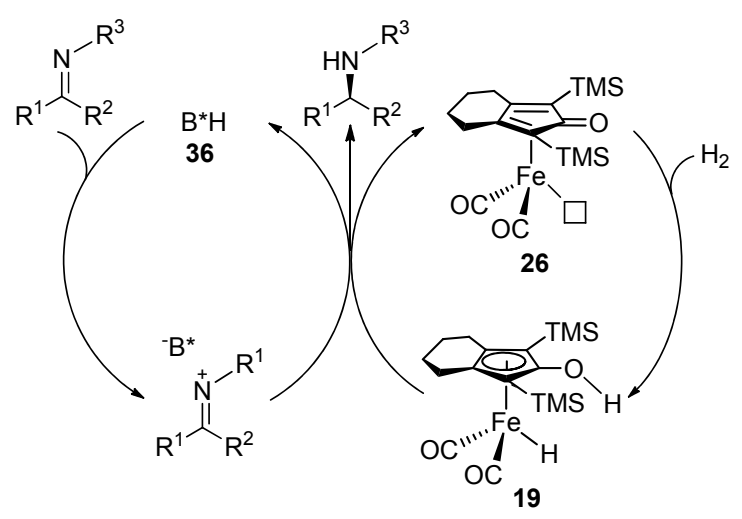

图式 16 铁络合物 19 和手性磷酸协同催化酮亚胺的不对称氢 化反应

Scheme 16 Asymmetric hydrogenation of ketoimines catalyzed by iron complex 19 and chiral phosphoric acid

后来, Beller 等 ${ }^{[46]}$ 还将相同的策略用于环状亚胺 (Eq. 22)以及含有亚胺结构的喹喔啉(Eq. 23)的不对称氢 化反应中, 在与链状亚胺相似的反应条件下, 获得了中 等或较高的对映选择性.

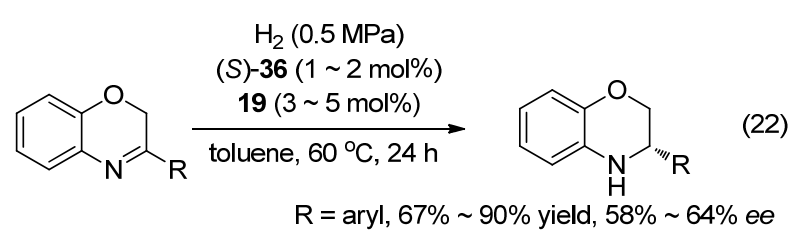




$$
\begin{aligned}
& \mathrm{H}_{2}(0.5 \mathrm{MPa})
\end{aligned}
$$

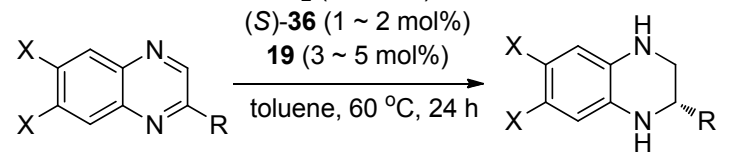

$$
\begin{aligned}
& \mathrm{X}=\mathrm{H}, \mathrm{R}=\text { aryl; } \quad 83 \% \sim 95 \% \text { yield, } 60 \% \sim 94 \% \text { ee } \\
& \mathrm{X}=\mathrm{H}, \mathrm{R}=\text { alkyl; } 94 \% \text { yield, } 56 \% \sim 66 \% \text { ee } \\
& \mathrm{X}=\mathrm{Me}, \mathrm{R}=\mathrm{Ph} ; 80 \% \text { yield, } 65 \% \text { ee }
\end{aligned}
$$

Beller 等 ${ }^{[47]}$ 还将上述协同催化体系用于串联反应中 (Scheme 17). 他们使用单膦配体和金的络合物 37 催化 端炔的氢胺化反应合成酮亚胺, 该酮亚胺不经分离, 在 铁催化剂 19 和手性磷酸 $(R)-36$ 协同催化下发生不对称 氢化反应，同样可以取得较高的对映选择性.

$$
\begin{aligned}
& \mathrm{R}^{1}=+\mathrm{R}^{2} \mathrm{NH}_{2} \quad \frac{37(1 \mathrm{~mol} \%)}{\text { toluene, r.t., } 16 \mathrm{~h}} \\
& \mathrm{H}_{2}(5.0 \mathrm{MPa})
\end{aligned}
$$

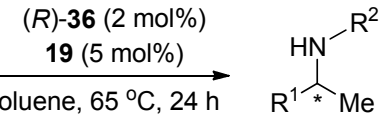

$$
\begin{aligned}
& 71 \% \sim 96 \% \text { yield } \\
& 70 \% \text { 94\% ee }
\end{aligned}
$$

图式 17 革联反应中亚胺的不对称催化氢化反应

Scheme 17 Catalytic asymmetric hydrogenation of imines in tandem reactions

\section{2 氮一膦多齿配体和铁的络合物催化剂}

2008 年, Morris 等 ${ }^{[48]}$ 将四齿膦-氮配体和二价铁络 合物 $(R, R)-38$ 用于苯乙酮的氢化反应中, 在 $50{ }^{\circ} \mathrm{C}, 2.53$ $\mathrm{MPa} \mathrm{H}_{2}$ 压力下, 得到 $40 \%$ 的转化率和 $27 \%$ ee 的对映选 择性(Eq. 24). 应当指出的是, 这类催化剂在酮的转移氢 化中也表现出优异的催化性能 ${ }^{[7 \mathrm{e}]}$. 在反应中, 亚胺配体 和胺基配体给出相似的反应性, 说明这两类催化剂在反 应中可能生成了相同的含有 H-Fe-N-H 结构的催化剂, 通过球外协同机理完成了氢化反应(参见 Eq. 15).

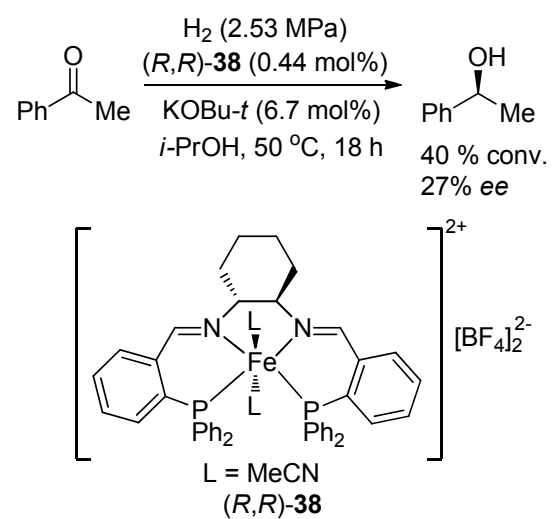

最近 Morris 等 ${ }^{[49]}$ 又发展了一类三齿的手性膦-氮配 体和铁的络合物催化剂, 高效地实现了多种酮的氢化反 应, TON 最高达到 5000, TOF 最高达 $2000 \mathrm{~h}^{-1}$. 当使用手 性络合物 $(S, S)-39$ 作为催化剂时，能获得最高 $85 \% \mathrm{ee}$ 的 对映选择性(表 5). 和其它手性金属络合物类似，该催化 剂主要对芳基烷基酮比较有效, 而且随着烷基部分的位 阻增加, 反应的对映选择性降低; 而脂肪酮氢化的对映 选择性一般很低.

\section{表 5 铁络合物 $(S, S)$-39 催化酮的不对称氢化反应}

Table 5 Asymmetric hydrogenation of ketones catalyzed by iron complex $(S, S)-\mathbf{3 9}$

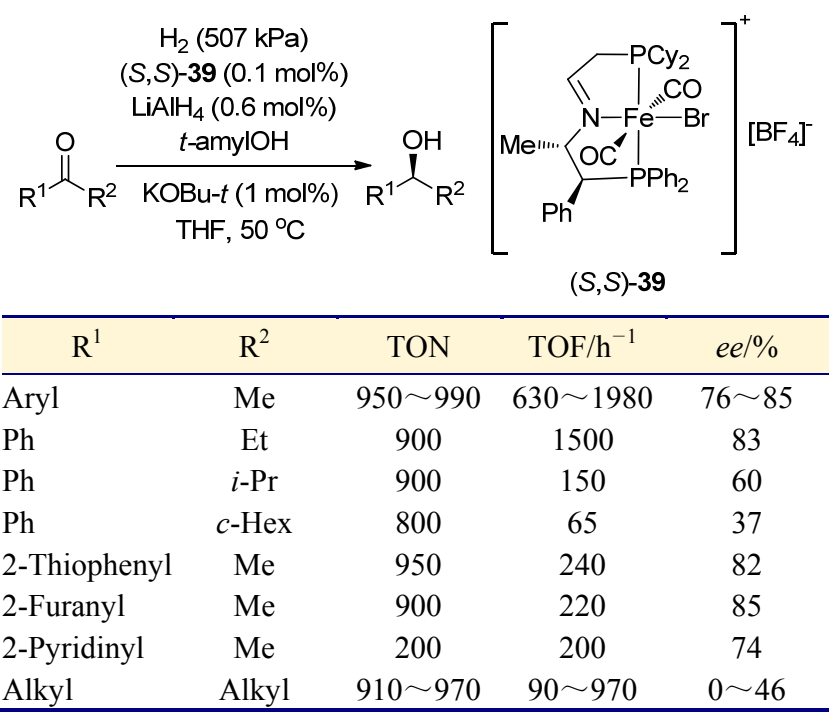

2011 年, Milstein 等 ${ }^{[50]}$ 合成了一类钳式配体和铁的 络合物 40, 并成功将其应用于醛、酮的氢化反应中(Eq. 25). 该催化剂具有比较高的活性, 在常温, $415 \mathrm{kPa} \mathrm{H}_{2}$ 下即可完成氢化, TON 最高达到 1880 .
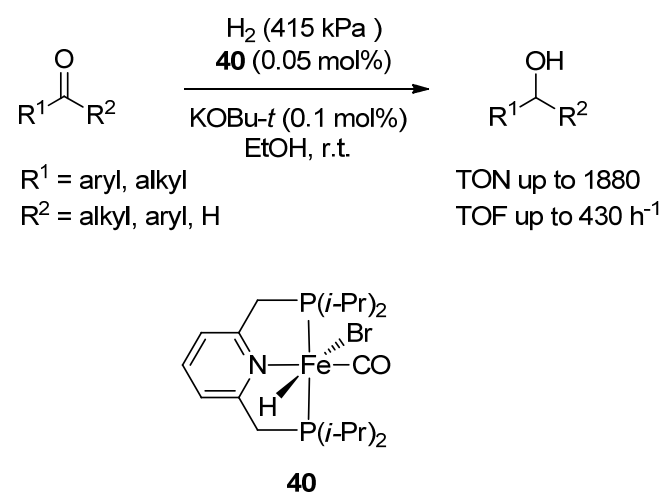

后来, Milstein 等 ${ }^{[51]}$ 将 $\mathbf{4 0}$ 与 $\mathrm{NaBH}_{4}$ 和 $\mathrm{EtOH}$ 反应, 合 成了一种新的铁络合物 41 (Scheme 18). 该络合物不需 要碱性添加剂, 就能够催化醛和酮的氢化反应, TON 最 高达 1980 . 


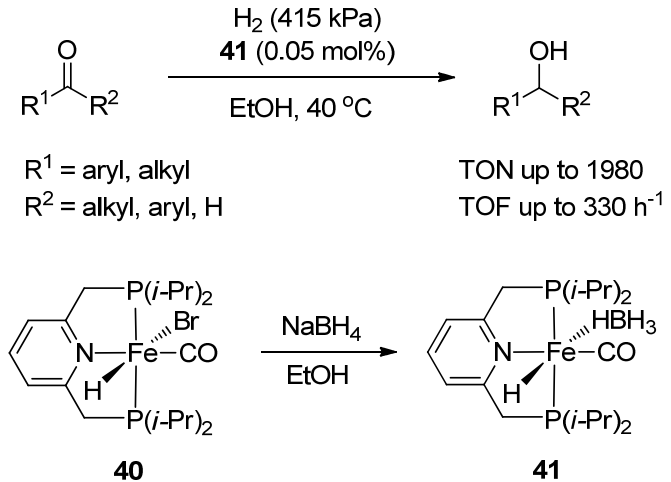

图式 18 铁络合物 41 催化醛和酮的氢化反应

Scheme 18 Hydrogenation of aldehydes and ketones catalyzed by iron complex 41

Milstein 等对上述氢化反应提出了如下可能机理: 催化剂 40 首先在碱存在条件下脱去 $\mathrm{KBr}$, 然后和乙醇 反应脱氢, 得到去芳构化的中间体 42 . 络合物 41 也可 以在乙醇促进下经过连续脱氢, 得到中间体 42. 氢气在 络合物 42 作用下发生异裂, 得到零价铁中间体 43 . 中 间体 43 通过一种新颖的外围氢转移的过渡态 44 , 把醇 上的质子和配体上的氢分别转移给羰基的氧和碳, 生成 氢化产物, 并完成催化剂的再生(Scheme 19).

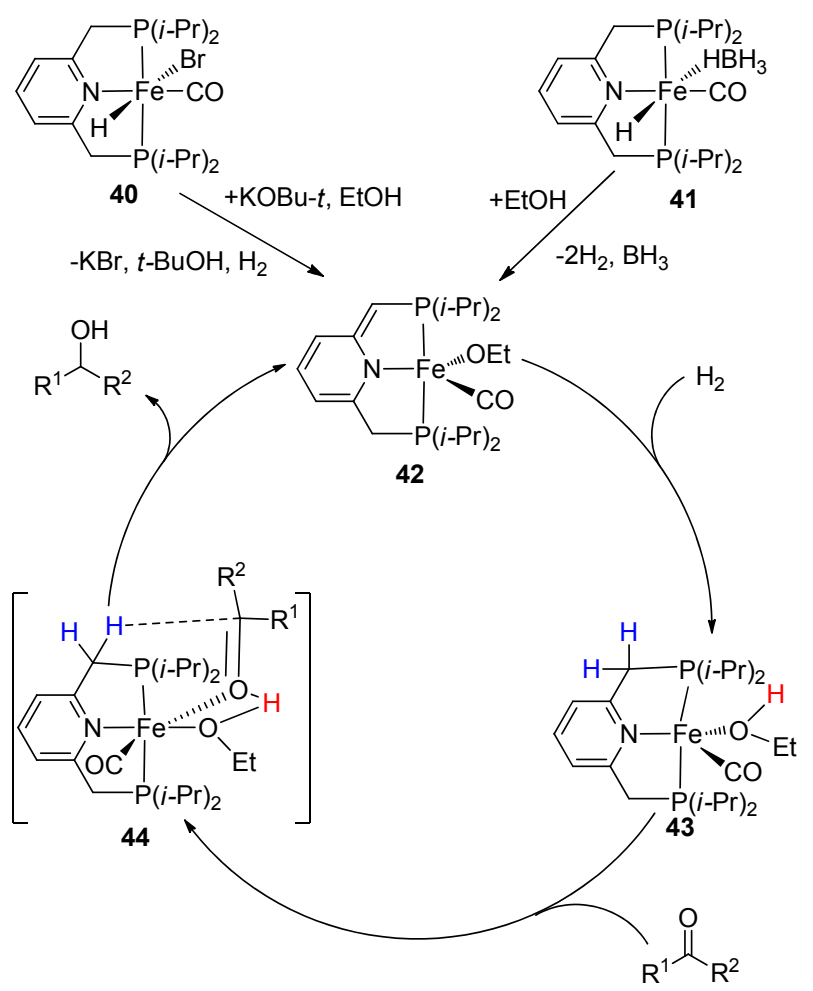

图式 19 铁络合物 43 和 44 催化羰基化合物氢化的可能机理 Scheme 19 Proposed mechanism of hydrogenation of carbonyl compounds catalyzed by iron complex 43 or $\mathbf{4 4}$

最近, Milstein 等 ${ }^{[52]}$ 又使用钳式配体和铁的负氢络 合物 45 实现了三氟乙酸酯的氢化反应, 得到三氟乙醇
(Eq. 26). 该反应的历程和酮的氢化类似, 首先将三氟乙 酸酯氢化到三氟乙醛, 然后再经历一个催化循环, 得到 最终氢化产物.

$$
\text { R=alkyl }
$$

Fairweather 和 Guan ${ }^{[53 \mathrm{a}]}$ 以及 Beller 等 ${ }^{[53 \mathrm{~b}]}$ 几乎同时报 道了使用通过脂肪胺连接的 P-N-P 三齿配体和铁的络合 物 46, 实现了多种非活化链状酯类化合物的氢化反应 (Eq. 27). Fairweather 和 Guan 等还成功将该催化剂用于 饱和脂肪酸酯的氢化中.

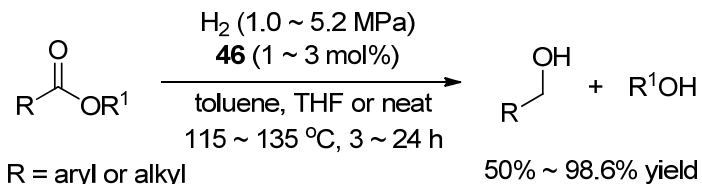

$$
\begin{aligned}
& \begin{array}{l}
\mathrm{R}=\text { aryl or alkyl } \\
\mathrm{R}^{1}=\mathrm{Me}
\end{array} \\
& \text { (Pr-i) })_{46}^{-\mathrm{H}_{2}}
\end{aligned}
$$

\section{3 多齿膦配体和铁的负氢络合物催化剂}

2013 年, Beller 等 ${ }^{[54]}$ 使用四齿膦配体和铁的络合物 47 催化 $\alpha, \beta$-不饱和醛的氢化反应, 获得了很好的化学选 择性, 得到系列烯丙醇产物(Eq. 28). 该催化剂也可以催 化简单醛的氢化，同样获得比较好的结果. 和前面介绍 的催化剂 25 (Eq. 20)不同，该催化剂在酸性条件下实现 氢化反应，而且其所需要的反应温度比较高.

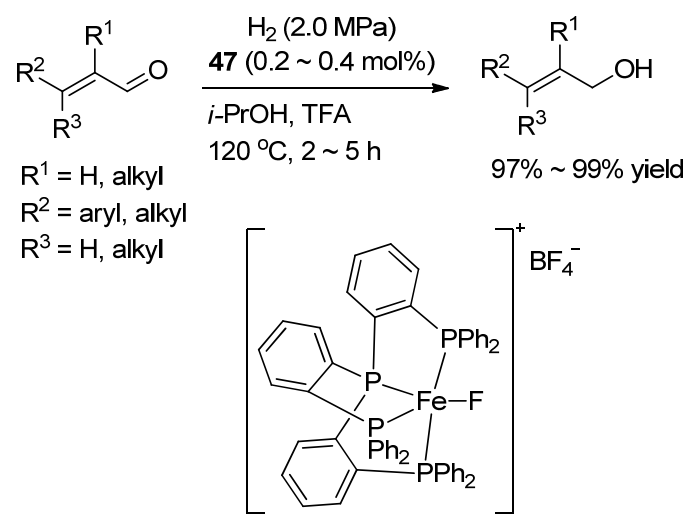


根据核磁实验和理论计算的结果, Beller 等 ${ }^{[54]}$ 提出 了下列催化机理(Scheme 20): 催化剂 47 首先和氢气作 用，产生铁负氢中间体 $\mathbf{4 8}$, 同时生成 HF. 该负氢中间体 48 在质子促进下解离一个配位的膦原子, 生成配位不 饱和的中间体 49 . 中间体 49 和底物配位后发生负氢转 移, 得到中间体 $\mathbf{5 0}$, 最后通过氢气异裂, 生成氢化产物, 同时释放质子, 实现催化剂的再生. 在催化循环中, 质 子酸能够显著降低配体解离步骤的能垒.

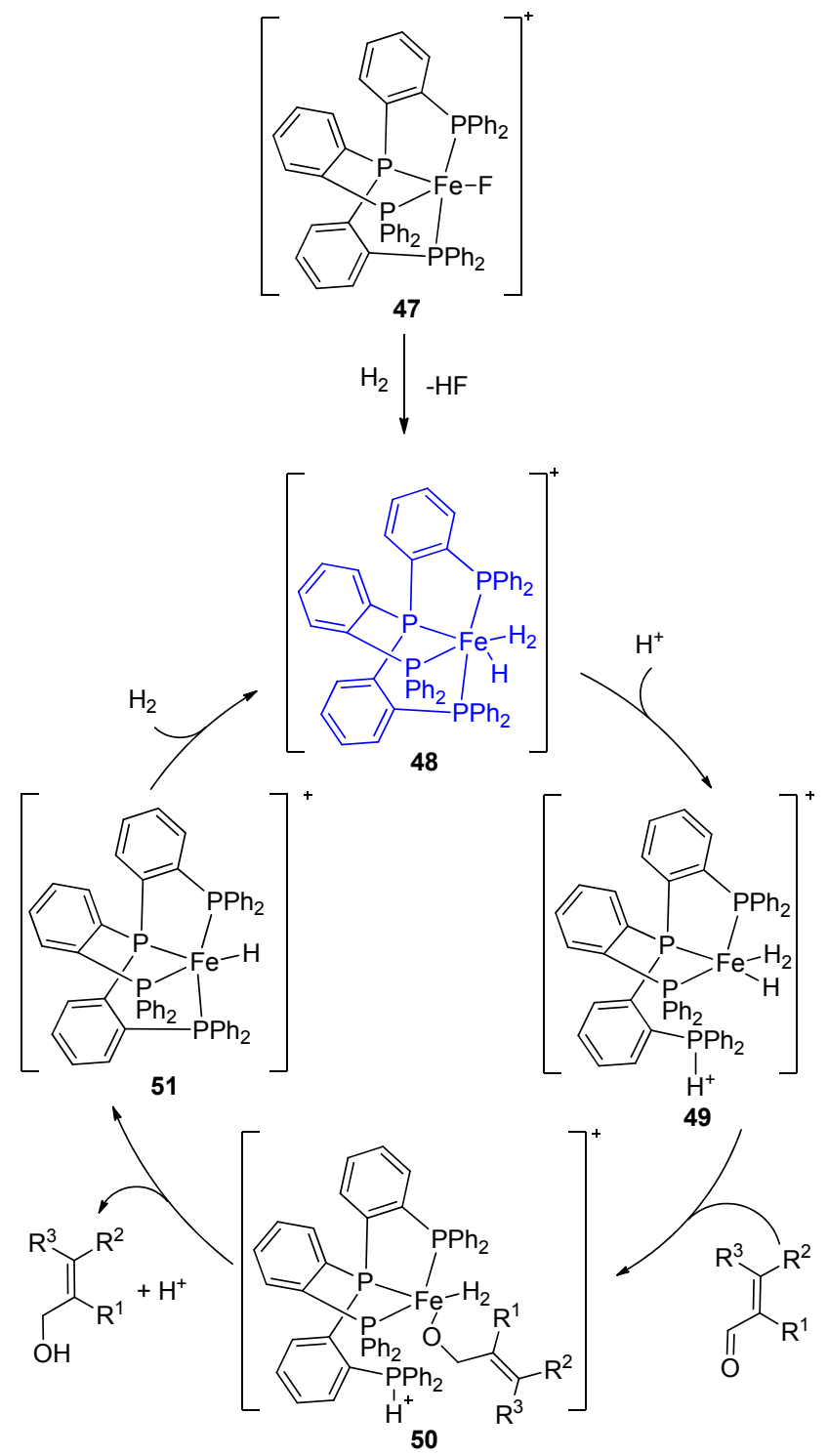

图式 20 铁络合物 47 催化 $\alpha, \beta$-不饱和醛氢化反应的可能机理 Scheme 20 Proposed mechanism of the hydrogenation of $\alpha, \beta$-unsaturated aldehydes catalyzed by iron complex $\mathbf{4 7}$

\section{4 非均相催化剂}

最近, 肖建良和高景星等 ${ }^{[55]}$ 使用具有 20 元环结构 的手性 PN 配体 $\mathbf{5 2}$ 和 $\mathrm{Fe}_{3}(\mathrm{CO})_{12}$ 结合作为催化剂, 实现 了简单酮和 $\beta$-酮酸酯的高对映选择性氢化(Scheme 21).
该体系底物适用范围很广，对于各类芳基烷基酮以及 $\beta$ 芳基取代的 $\beta$-酮酸酯都能给出很高的对映选择性. 这些 结果代表铁催化酮的不对称氢化反应目前最好水平. 机 理研究表明, 该催化体系很可能是非均相体系, 被手性 配体 $(R, R, R, R)-\mathbf{5 2}$ 修饰的纳米铁颗粒是真正的手性催化 剂，它能够高效地实现酮的不对称氢化反应。该研究还 对发展非均相的手性铁催化剂具有很好的借鉴作用.

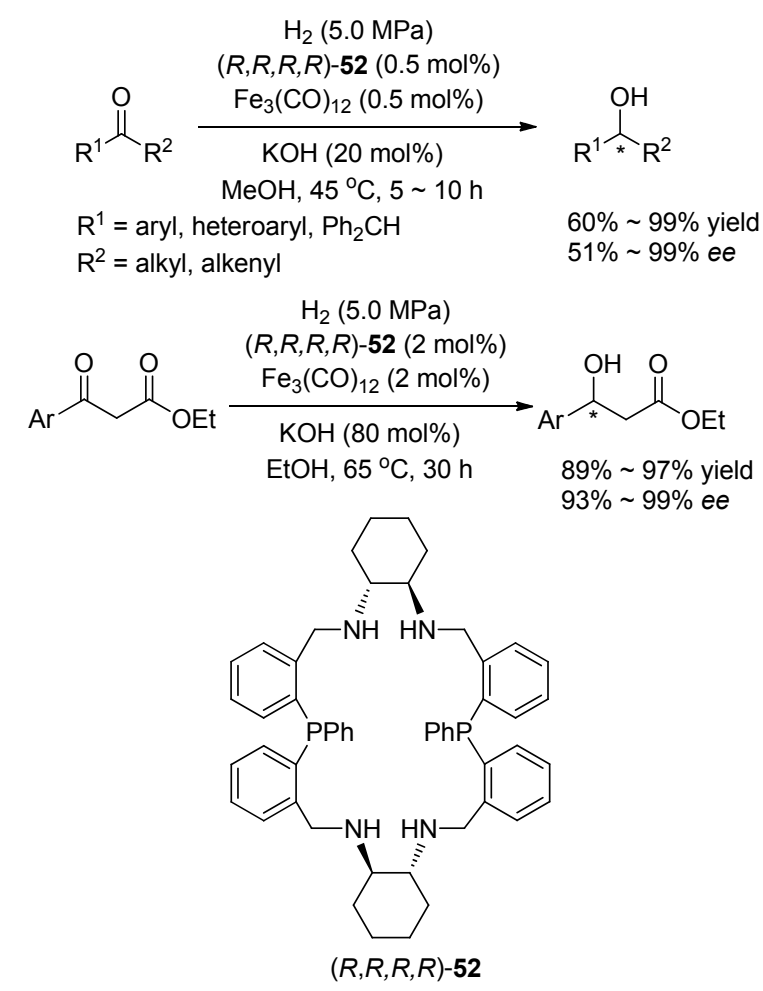

图式 $21 \mathrm{Fe} / \mathbf{5 2}$ 催化酮的不对称氢化反应

Scheme 21 Asymmetric hydrogenation of ketones catalyzed by iron $/ \mathbf{5 2}$

\section{3 结论与展望}

虽然铁催化合成氨(Haber-Bosch 工艺)是催化反应 的典范, 也是世界上最重要的催化反应, 而且均相铁催 化氢化反应与 Wilkinson 催化剂几乎在同一时期被发现, 但是在 Wilkinson 催化剂迅猛发展的 40 余年里, 铁催化 的氢化反应几乎完全被人们忽略. 在 2004 年, Bolm 等 ${ }^{[7 \mathrm{~d}]}$ 在有关铁催化有机反应的的综述文章中，仅仅用 了短短的一段话介绍铁催化的氢化反应. 有趣的是, 同 样是在 2004 年, Chirik 等 ${ }^{[14 a]}$ 报道了吡啶亚胺配体和铁的 络合物 7 催化烯烃的氢化反应, 其活性甚至超过了 Wilkinson 催化剂. 这一研究使得人们重新审视铁催化 氢化的可能性. 最近, 随着人们对可持续发展化学以及 绿色化学的关注不断增强, 铁催化的氢化反应才重新受 到重视，成为一个非常活跃的研究领域. 
在过去的 10 年里, 有关铁催化氢化反应的研究不 断取得突破, 新的铁催化剂不断被开发出来, 深刻影响 了催化氢化领域. 除了 Chirik 发展的用于烯烃氢化的催 化剂 7 以外, 一系列双功能铁催化剂也被发展出来, 并 成功应用于酫、酮和亚胺的氢化反应中, 催化效率也不 断被刷新. 目前在一些底物的氢化中其结果已经可以和 贵金属催化剂相媲美. 在催化剂设计方面, 从最初简单 模仿贵金属催化剂, 到不断发展新的催化模式, 比如双 自由基结构的催化剂 $7^{[14]}$ 和具有大环结构的配体与纳米 铁组成的手性非均相催化剂 ${ }^{[55]}$, 都是非常新颖的. 这 些进展充分显示了铁催化氢化的巨大潜力.

但是还应看到，和传统的贵金属催化剂相比，现有 的铁催化剂无论从活性、选择性、官能团耐受性还是催 化剂的稳定性方面, 都存在很大差距. 比如对于铁催化 烯烃的氢化而言, 至今还没有实现其不对称转化 ${ }^{[56]}$; 再 如, 对于酮的氢化反应, 贵金属催化剂已经可以达到数 百万的 TON 和数十万的 TOF, 同时还能给出极高的对 映选择性 ${ }^{[57]}$, 但是铁催化剂目前的记录都在 5000 以内. 这些巨大的差距都需要发展新的铁催化剂和催化模式 来弥补. 我们有理由相信, 铁催化氢化反应将会得到越 来越广泛的应用 ${ }^{[58]}$.

\section{References}

[1] (a) de Vries, J. G.; Elsevier, C. J. The Handbook of Homogeneous Hydrogenation, Wiley-VCH, Weinheim, 2007.

(b) Gallezot, P. Hydrogenation - Heterogeneous in Encyclopedia of Catalysis, Vol. 4, Ed.: Horvath, I. T., Wiley, Hoboken, 2003.

[2] Osborn, J. A.; Jardine, F. H.; Young, J. F.; Wilkinson, G. J. Chem. Soc. A 1966, 1711.

[3] (a) Jacobsen, E. N.; Pfaltz, A.; Yamamoto, H. Comprehensive Asymmetric Catalysis, Springer, Berlin, 1999.

(b) Tang, W.; Zhang, X. Chem. Rev. 2003, 103, 3029.

(c) Xie, J. H.; Zhou, Q. L. Acta Chim. Sinica 2012, 70, 1427 (in Chinese).

(谢建华, 周其林, 化学学报, 2012, 70, 1427.)

[4] (a) Knowles, W. S. Angew. Chem., Int. Ed. 2002, 41, 1998.

(b) Noyori, R. Angew. Chem., Int. Ed. 2002, 41, 2008.

[5] (a) Blaser, H.-U.; Schmidt, E. Asymmetric Catalysis on Industrial Scale: Challenges, Approaches and Solutions, Wiley-VCH, Weinheim, Germany, 2004.

(b) Shimizu, H.; Nagasaki, I.; Matsumura, K.; Sayo, N.; Saito, T. Acc. Chem. Res. 2007, 40, 1385.

(c) Johnson, N. B.; Lennon, I. C.; Moran, P. H.; Ramsden, J. A. Acc. Chem. Res. 2007, 40, 1291.

(d) Ager, D. J.; de Vries, A. H. M.; de Vires, J. G. Chem. Soc. Rev. 2012, 41, 3340 .

[6] For selected reviews, see: (a) Enthaler, S.; Junge, K.; Beller, M. Angew. Chem., Int. Ed. 2008, 47, 3317.

(b) Nakamura, E.; Yoshikai, N. J. Org. Chem. 2010, 75, 6061.

(c) Sun, C.-L.; Li, B.-J.; Shi, Z.-J. Chem. Rev. 2011, 111, 1293.

(d) Zhu, S.-F.; Zhou, Q.-L. Nat. Sci. Rev. 2014, 1, 580.

(e) Xu, D.; Xiao, W.; Peng, J.; Li, J.; Bai, Y. Chin. J. Org. Chem. 2014, 34, 2195 (in Chinese).

(徐大鹏，肖文军，彭家建，历嘉云，白赢，有机化学，2014，34,
2195.)

For selected examples, see:: (f) Zhu, S.-F.; Cai, Y.; Mao, H.-X.; Xie, J.-H.; Zhou, Q.-L. Nat. Chem. 2010, 2, 546.

(g) Cai, Y.; Zhu, S.-F.; Wang, G.-P.; Zhou, Q.-L. Adv. Synth. Catal. 2011, 353, 2939.

(h) Tondreau, A. M.; Atienza, C. C. H.; Weller, K. J.; Nye, S. A.; Lewis, K. M.; Delis, J. G. P.; Chirik, P. J. Science 2012, 335, 567.

(i) Guo, X.; Fang, G.; Li, G.; Ma, H.; Fan, H.; Yu, L.; Ma, C.; Wu, X.; Deng, D.; Wei, M.; Tan, D.; Si, R.; Zhang, S.; Li, J.; Sun, L.; Tang, Z.; Pan, X.; Bao, X. Science 2014, 344, 616.

(j) Shen, J.-J.; Zhu, S.-F.; Cai, Y.; Xu, H.; Xie, X.-L.; Zhou, Q.-L. Angew. Chem., Int. Ed. 2014, 53, 13188.

(k) Zhang, Y.; Luo, S.; Feng, B.-N. Chin. J. Org. Chem. 2014, 34, 2249 (in Chinese).

(张艳, 罗莎, 冯柏年, 有机化学, 2014, 34, 2249.)

(1) Liu, S.-S.; Jiang, K.; Pi, D.-W.; Zhou, H.-F.; Uozumi, Y.; Zou, K. Chin. J. Org. Chem. 2014, 34, 1369 (in Chinese).

(刘森生, 姜坤, 皮单违, 周海峰, Uozumi, Y., 邹坤, 有机化学, 2014, 34, 1369.)

[7] (a) Enthaler, S.; Junge, K.; Beller, M. In Iron Catalysis in Organic Chemistry-Reactions and Applications, Vol. 4, Ed.: Plietker, B., Wiley, Weinheim, 2008.

(b) Chirik, P. J. Modern Alchemy: Replacing Precious Metals with Iron in Catalytic Alkene and Carbonyl Hydrogenation Reactions in Catalysis without Precious Metals, Ed.: Bullock, R. M., Wiley-VCH, Weinheim, 2010, Chapter 4.

(c) Nakazawa, H.; Itazaki, M. Fe-H Complexes in Catalysis in Iron Catalysis - Fundamentals and Applications, Ed.: Plietker, B., Springer, Heideberg, 2011.

(d) Bolm, C.; Legros, J.; Le Paih, J.; Zan, L. Chem. Rev. 2004, 104, 6217.

(e) Morris, R. H. Chem. Soc. Rev. 2009, 38, 2282.

(f) Junge, K.; Schröder, K.; Beller, M. Chem. Commun. 2011, 47, 4849.

[8] Frankel, E. N.; Emken, E. A.; Peters, H. M.; Davison, V. L.; Butterfield, R. O. J. Org. Chem. 1964, 29, 3292.

[9] Frankel, E. N.; Emken, E. A.; Davison, V. L. J. Org. Chem. 1965, 30, 2739.

[10] Cais, M.; Maoz, N. J. Chem. Soc. A 1971, 1811.

[11] Schroeder, M. A.; Wrighton, M. S. J. Am. Chem. Soc. 1976, 98 , 551.

[12] (a) Weiller, B. H.; Miller, M. E.; Grant, E. R. J. Am. Chem. Soc. 1987, 109, 352 .

(b) Weiller, B. H.; Grant, E. R. J. Am. Chem. Soc. 1987, 109, 1051. (c) Miller, M. E.; Grant, E. R. J. Am. Chem. Soc. 1987, 109, 7951.

[13] Small, B. L.; Brookhart, M.; Bennett, A. M. A. J. Am. Chem. Soc. 1998, 120, 4049.

[14] (a) Bart, S. C.; Lobkovsky, E.; Chirik, P. J. J. Am. Chem. Soc. 2004, 126, 13794.

(b) Bart, S. C.; Lobkovsky, E.; Bill, E.; Wieghardt, K.; Chirik, P. J. Inorg. Chem. 2007, 46, 7055.

(c) Trovitch, R. J.; Lobkovsky, E.; Bill, E.; Chirik, P. J. Organometallics 2008, 27, 1470.

(d) Russell, S. K.; Darmon, J. M.; Lobkovsky, E.; Chirik, P. J. Inorg. Chem. 2010, 49, 2782.

[15] Bart, S. C.; Chlopek, K.; Bill, E.; Bouwkamp, M. W.; Lobkovsky, E.; Neese, F.; Wieghardt, K.; Chirik, P. J. J. Am. Chem. Soc. 2006, 128, 13901.

[16] Archer, A. M.; Bouwkamp, M. W.; Cortez, M.-P.; Lobkovsky, E.; Chirik, P. J. Organometallics 2006, 25, 4269.

[17] Bart, S. C.; Hawrelak, E. J.; Lobkovsky, E.; Chirik, P. J. Organometallics 2005, 24, 5518 . 
[18] Trovitch, R. J.; Lobkovsky, E.; Chirik, P. J. Inorg. Chem. 2006, 45, 7252.

[19] Yu, R. P.; Darmon, J. M.; Hoyt, J. M.; Margulieux, G. W.; Turner, Z. R.; Chirik, P. J. ACS Catal. 2012, 2, 1760.

[20] (a) Bianchini, C.; Meli, A.; Peruzzini, M.; Vizza, F.; Zanobini, F. Organometallics 1989, 8, 2080.

(b) Bianchini, C.; Meli, A.; Peruzzini, M.; Frediani, P.; Bohanna, C.; Esteruelas, M. A.; Ora, L. A. Organomentallics 1992, 11, 138.

[21] Daida, E. J.; Peters, J. C. Inorg. Chem. 2004, 43, 7474.

[22] Fong, H.; Moret, M.-E.; Lee, Y.; Peters, J. C. Organometallics 2013, 32, 3053.

[23] Srimani, D.; Diskin-Posner, Y.; Ben-David, Y.; Milstein, D. Angew. Chem., Int. Ed. 2013, 52, 14131.

[24] Tajima, Y.; Kunioka, E. J. Org. Chem. 1968, 33, 1689.

[25] (a) Inoue, H.; Suzuki, M. J. Chem. Soc., Chem. Commun. 1980, 817.

(b) Inoue, H.; Sato, M. J. Chem. Soc., Chem. Commun. 1983, 983.

[26] Frank, D. J.; Guiet, L.; Käslin, A.; Murphy, E.; Thomas, S. P. RSC Adv. 2013, 3, 25698.

[27] Phua, P.-H.; Lefort, L.; Boogers, J. A. F.; Tristany, M.; de Vries, J. G. Chem. Commun. 2009, 3747.

[28] Rangheard, C.; de Julián Fernández, C.; Phua, P.-H.; Hoorn, J.; Lefort, L.; de Vries, J. G. Dalton Trans. 2010, 39, 8464.

[29] Welther, A.; Bauer, M.; Mayer, M.; von Wangelin, A. J. ChemCatChem 2012, 4, 1088.

[30] Gieshoff, T. N.; Welther, A.; Kessler, M. T.; Prechtl, M. H. G.; von Wangelin, A. J. Chem. Commun. 2014, 50, 2261.

[31] Stein, M.; Wieland, J.; Steurer, P.; Tölle, F.; Mülhaupt, R.; Breit, B. Adv. Synth. Catal. 2011, 353, 523.

[32] Kelsen, V.; Wendt, B.; Werkmeister, S.; Junge, K.; Beller M.; Chaudret, B. Chem. Commun. 2013, 49, 3416.

[33] Hudson, R.; Rivière, A.; Cirtiu, C. M.; Luska, K. L.; Moores, A. Chem. Commun. 2012, 48, 3360.

[34] For a review, see: Bauer, G.; Kirchner, K. A. Angew. Chem., Int. Ed. 2011, 50, 5798.

[35] Knölker, H.-J.; Baum, E.; Goesmann, H.; Klauss, R. Angew. Chem., Int. Ed. 1999, 38, 2064

[36] (a) Blum, Y.; Czarkie, D.; Rahamim, Y.; Shvo, Y. Organometallics 1985, 4, 1459

(b) Shvo, Y.; Czarkie, D.; Rahamim Y.; Chodosh, D. F. J. Am. Chem. Soc. 1986, 108, 7400.

[37] (a) Casey, C. P.; Guan, H. J. Am. Chem. Soc. 2007, 129, 5816. For selected reviews, see: (b) Bullock, R. M. Angew. Chem., Int. Ed. 2007, 46, 7360.

(c) Quintard, A.; Rodriguez, J. Angew. Chem., Int. Ed. 2014, 53, 4404.

[38] (a) Casey, C. P.; Guan, H. J. Am. Chem. Soc. 2009, 131, 2499. For a review, see: (b) Chakraborty, S.; Guan, H. Dalton Trans. 2010, 39, 7427

[39] Zhang, H.-H.; Chen, D.-Z.; Zhang, Y.-H.; Zhang, G.-Q.; Liu, J.-B. Dalton Trans. 2010, 39, 1972

[40] Lu, X.; Zhang, Y.-W.; Yun, P.; Zhang, M.-T.; Li, T.-L. Org. Biomol. Chem. 2013, 11, 5264.

[41] (a) Pagnoux-Ozherelyeva, A.; Pannetier, N.; Mbaye, M. D.; Gaillard, S.; Renaud, J.-L. Angew. Chem. Int. Ed. 2012, 51, 4976.

(b) Moulin, S.; Dentel, H.; Pagnoux-Ozherelyeva, A.; Gaillard, S.;
Poater, A.; Cavallo, L.; Lohier, J.-F.; Renaud, J.-L. Chem. Eur. J. 2013, 19, 17881

[42] Mérel, D. S.; Elie, M.; Lohier, J.-F.; Gaillard, S.; Renaud, J.-L. ChemCatChem 2013, 5, 2939.

[43] Fleischer, S.; Zhou, S.-L.; Junge, K.; Beller, M. Angew. Chem., Int. Ed. 2013, 52, 5120.

[44] Berkessel, A.; Reichau, S.; von der Höh, A.; Leconte, N.; Neudörfl, J.-M. Organometallics 2011, 30, 3880.

[45] Zhou, S.; Fleischer, S.; Junge, K.; Beller, M. Angew. Chem., Int. Ed. 2011, 50, 5120.

[46] Fleischer, S.; Zhou, S.; Werkmeister, S.; Junge, K.; Beller, M. Chem. Eur. J. 2013, 19, 4997.

[47] Fleischer, S.; Werkmeister, S.; Zhou, S.; Junge, K.; Beller, M. Chem. Eur. J. 2012, 18, 9005.

[48] (a) Sui-Seng, C.; Freutel, F.; Lough, A. J.; Morris, R. H. Angew. Chem., Int. Ed. 2008, 47, 940.

(b) Sui-Seng, C.; Haque, F. N.; Hadzovic, A.; Pütz, A.-M.; Reuss, V.; Meyer, N.; Lough, A. J.; Zimmer-De Iuliis, M.; Morris, R. H. Inorg. Chem. 2009, 48, 735.

[49] Lagaditis, P. O.; Sues, P. E.; Sonnenberg, J. F.; Wang, K. Y.; Lough, A. J.; Morris, R. H. J. Am. Chem. Soc. 2014, 136, 1367.

[50] Langer, R.; Leitus, G.; Ben-David, Y.; Milstein, D. Angew. Chem., Int. Ed. 2011, 50, 2120.

[51] Langer, R.; Iron, M. A.; Konstantinovski, L.; Diskin-Posner, Y.; Leitus, G.; Ben-David, Y.; Milstein, D. Chem. Eur. J. 2012, 18, 7196.

[52] Zell, T.; Ben-David, Y.; Milstein, D. Angew. Chem., Int. Ed. 2014, 53,4685 .

[53] (a) Chakraborty, S.; Dai, H.-G.; Bhattacharya, P.; Fairweather, N. T.; Gibson, M. S.; Krause, J. A.; Guan, H.-R. J. Am. Chem. Soc. 2014, 136, 7869 .

(b) Werkmeister, S.; Junge, K.; Wendt, B.; Alberico, E.; Jiao, H.; Baumann, W.; Junge, H.; Gallou, F.; Beller, M. Angew. Chem., Int. Ed. 2014, 53, 8722 .

[54] Wienhöfer, G.; Westerhaus, F. A.; Junge, K.; Ludwig, R.; Beller, M. Chem. Eur. J. 2013, 19, 7701

[55] Li, Y.-Y.; Yu, S.-L.; Wu, X.-F.; Xiao, J.-L.; Shen, W.-Y.; Dong, Z.-R.; Gao, J.-X. J. Am. Chem. Soc. 2014, 136, 4031.

[56] Hoyt, J. M.; Shevlin, M.; Margulieux, G. W.; Krska, S. W.; Tudge, M. T.; Chirik, P. J. Organometallics 2014, 33, 5781.

[57] Xie, J.-H.; Liu, X.-Y.; Xie, J.-B.; Wang, L.-X.; Zhou, Q.-L. Angew. Chem., Int. Ed. 2011, 50, 7329.

[58] After the acceptance of this paper, several significant progresses in iron-catalyzed hydrogenation was reported. For hydrogenation of olefins, see: (a) Gärtner, D.; Welther, A.; Rad, B. R.; Wolf, R.; von Wangelin, A. J. Angew. Chem., Int. Ed. 2014, 53, 3722.

(b) Manna, K.; Zhang, T.; Carboni, M.; Abney, C. W.; Lin, W. J. Am. Chem. Soc. 2014, 136, 13182.

(c) Guo, N.; Hu, M.-Y.; Feng, Y.; Zhu, S.-F. Org. Chem. Front. 2015, 2, 692 .

For hydrogenation of carbonyl compounds and imines, see: (d) Gorgas, N.; Stöger, B.; Veiros, L. F.; Pittenauer, E.; Allmaier, G.; Kirchner, K. Organometallics 2014, 33, 6905.

(e) Lu, L.-Q.; Li, Y.; Junge, K.; Beller, M. J. Am. Chem. Soc. 2015 $137,2763$. 